\title{
Refining the chronology of Acheulean deposits at Porto Maior in the River Miño basin (Galicia, Spain) using a comparative luminescence and ESR dating approach
}

\author{
Martina Demuro ${ }^{1 *}$, Lee J. Arnold ${ }^{1}$, Mathieu Duval ${ }^{2,3}$, Eduardo Méndez-Quintas ${ }^{3}$, Manuel Santonja ${ }^{3}$, Alfredo \\ Pérez-González ${ }^{4}$
}

\begin{abstract}
1 School of Physical Sciences, Environment Institute, and Institute for Photonics and Advanced Sensing (IPAS), University of Adelaide, North Terrace Campus, 5005 Adelaide, SA, Australia.

2 Australian Research Centre for Human Evolution (ARCHE), Environmental futures Research Institute, Griffith University, Nathan Campus, 4111, Brisbane, Queensland, Australia.

3 Centro Nacional de Investigación sobre la Evolución Humana (CENIEH), Paseo de Atapuerca, 3, 09002 Burgos, Spain.

4 Instituto de Evolución en África (IDEA),Covarrubias, 36, 28010 Madrid, Spain.
\end{abstract}

* Corresponding author, e-mail: martina.demuro@adelaide.edu.au

Keywords Single-grain TT-OSL dating, ESR dating, Bayesian modelling, fluvial archives, Acheulean, Porto Maior, Galicia

\begin{abstract}
Trapped charge dating of optically bleached quartz, which involves techniques such as electron spin resonance (ESR) and thermally transferred optically stimulated luminescence (TT-OSL), is increasingly being used to establish Middle and Early Pleistocene chronologies for archaeological, palaeontological and palaeoenvironmental sedimentary sequences. Comparative dating studies that make use of several independent or semi-independent radiometric techniques in tandem are an invaluable means of assessing the reliability of optically bleached quartz dating methods and ascertaining empirical relationships between different quartz dating signals. In this study, we undertake a comparative dating evaluation of single-grain TT-OSL and various ESR signals that exhibit different optical resetting properties (Al, Ti-Li and, where measurable, Ti-H centres) at the Middle Pleistocene archaeological site of Porto Maior, northwest Spain, which contains a distinctive, regionally significant Acheulean stone tool assemblage. We expand the existing chronological framework for Porto Maior using single-grain TT-OSL dating, derive combined numerical ages for individual depositional units using Bayesian modelling, and further examine the relationship between ESR and TT-OSL signals by reviewing a range of published comparative dating studies. The new single-grain TT-OSL ages obtained for unit PM3 at Porto Maior (225 $\pm 31 \mathrm{ka}$ and $242 \pm 32 \mathrm{ka}$ ) are in close agreement with previously published ESR Ti-Li ages for this unit ( $266 \pm 23 \mathrm{ka}$ and $279 \pm 26 \mathrm{ka})$ and significantly younger than those reported for the $\mathrm{Al}$ centre (441 $\pm 75 \mathrm{ka}$ and $452 \pm 62 \mathrm{ka}$ ). Our broader comparison of published TT-OSL - ESR dating results also shows statistically significant agreement between single-grain TT-OSL and Ti-Li centre ages in other sedimentary settings, suggesting a potentially closer relationship between the TT-OSL-producing traps and the Ti-Li paramagnetic centres in quartz, rather than between the TT-OSL and Al centre signals. The Bayesian modelling results for Porto Maior enable us to refine the existing bracketing depositional age for the archaeological accumulation at Porto Maior and provide a stronger statistical foundation for chronostratigraphic correlations with other Acheulean archaeological deposits found in the Miño River basin. The Bayesian model indicates that the entire Porto Maior sedimentary sequence was deposited between $312.6 \pm 32.6$ ka and $18.8 \pm$
\end{abstract}


7.7 ka, and that the in situ large cutting tool assemblage from level PM4 most likely accumulated during Marine Isotope Stage 7 (mean depositional age $=210.7 \pm 24.7 \mathrm{ka}$ ). The results of this study reinforce the usefulness of applying the multiple centre (MC) ESR dating approach in many dating contexts, and demonstrate the value of undertaking comparisons between semi-independent quartz dating signals in order to maximise the reliability of ESR and single-grain TT-OSL quartz chronologies.

\section{Introduction}

The last two decades have seen major advances in the development of trapped charge dating methods that can be applied to optically bleached sedimentary quartz from Early and Middle Pleistocene archaeological, palaeontological and palaeoenvironmental sites (Toyoda et al., 2000; Voinchet et al 2004; Wang et al., 2006; Duller and Wintle, 2012; Arnold et al., 2015; Duval et al., 2015; Wintle and Adamiec, 2017). In particular, quartz dating techniques such as electron spin resonance (ESR) and thermally transferred optically stimulated luminescence (TT-OSL) represent invaluable geochronology tools for a wide range of Quaternary research applications, including archaeological dating, as they (i) make use of ubiquitously preserved sedimentary materials (silicate minerals), (ii) cover an important timeframe in human evolution (>1 Ma $-100 \mathrm{ka})$ that is typically outside the range of alternative dating techniques (or for which the application of other dating methods is often not practical due to the absence of suitably preserved materials), and (iii) provide numerical age estimates instead of relative chronologies (unlike palaeomagnetism or biochronology).

ESR dating was first applied to optically bleached quartz during the mid-1980s (Yokoyama et al., 1985) but its use over the following two decades remained limited as ESR quartz dating applications continued to focus on volcanic and intra-fault contexts (see review by Grün, 1989). Methodological research undertaken during these early years did, however, pave the way for an improved general understanding of quartz ESR signals used in optically bleached dating contexts, particularly with regards to thermal stability considerations and optical bleaching kinetics (e.g. Toyoda and Ikeya, 1991; Falguères et al., 1991; Walther and Zilles, 1994). ESR dating of optically bleached quartz from Quaternary sedimentary deposits has traditionally focused on the use of the aluminium (Al) centre (e.g., Falguères, 1986; Laurent, 1993; Tanaka et al., 1995; Yoshida, 1996; Voinchet, 2002 and references therein), which is related to the most abundant trace element in quartz (Preusser et al., 2009) and gives rise to a strong ESR signal when measured at liquid nitrogen temperature. However, the relatively slow bleaching kinetics of the $\mathrm{Al}$ centre and the presence of a residual (optically unbleachable) $\mathrm{Al}$ signal component (Walther and Zilles, 1994) has led to increasing use of alternative quartz ESR dating signals, particularly titanium (Ti) centre signals in conjunction with Al centre signals (Toyoda et al., 2000), with encouraging results (e.g. Rink et al., 2007; Tissoux et al., 2008). Comparative chronological studies involving independent or semi-independent numerical dating methods (e.g., luminescence, Ar-Ar, combined Useries/ESR dating of fossil teeth) have also contributed to continued improvements in the reliability of ESR quartz dating methods over the last decade (e.g., Rink et al., 2007; Moreno et al., 2015; Arnold et al., 2014, 2015; Bartz et al., 2019; Duval et al., this volume). As a result, ESR dating of optically bleached quartz has now been reliably applied to a large number of archaeological records and associated terrestrial archives (typically fluvial deposits) from Europe (Voinchet et al 2015; Moreno et al., 2015; Duval et al., 2017; Pereira et al., 2018; 
Bahain et al., this volume; Duval et al., this volume), Asia-Oceania (Beerten et al., 2006; Liu et al., 2013; 2014; Ingicco et al., 2018) and northern Africa (Sahnouni et al., 2018), among other regions.

In parallel, there has been an increase in TT-OSL dating applications in archaeological and geomorphological contexts over the last decade (see review by Arnold et al. 2015). TT-OSL dating originally emerged as an alternative luminescence dating technique with the potential to extend the traditional upper age limit of quartz OSL (Wang et al., 2006). TT-OSL signals are measured after optically removing the conventional OSL signal and heating the sample (typically to $\geq 260^{\circ} \mathrm{C}$ ) to induce a transfer of charge from the TT-OSL electron trap into the main OSL dating trap (Wintle and Duller, 2012). The dose saturation limits of the TT-OSL signal is typically an order of magnitude higher than for conventional OSL and at least double that reported for pIRIRSL (Arnold et al., 2015), permitting finite age determination over Middle Pleistocene timescales (e.g., Jacobs et al., 2011; Arnold et al., 2014). Several different TT-OSL dating protocols have been developed to enable burial dose estimation using individual aliquots composed of hundreds or thousands of quartz grains (e.g., Wang et al., 2007; Tsukamoto et al., 2008; Porat et al., 2009; Stevens et al., 2009; Adamiec et al., 2010; Jacobs et al., 2011). Recent developments in TT-OSL research have seen the emergence of single-grain dating approaches (e.g., Arnold et al., 2013, 2014; Demuro et al., 2015), which offer a number of potential advantages over traditional multi-grain TT-OSL approaches for quartz samples with inherently bright luminescence signals. Such benefits include the ability to isolate grains with favourable dating properties (e.g., high dose saturation limits, charge transfer into 'fast' component OSL traps), the detection of inter-grain differences in problematic TT-OSL behaviours (including low thermal stabilities), and a means of circumventing averaging effects arising from simultaneously measuring grains with different bleaching histories, signal compositions or TT-OSL source trap properties (Arnold et al., 2014; Arnold and Demuro, 2015; Arnold et al. 2019).

The successful application of both ESR and TT-OSL dating methods depends on adequate signal bleaching by sunlight prior to the burial event being dated. This is an important consideration in any dating study because the resetting of ESR and TT-OSL signal down to low or constant residual levels has been shown to require up to several weeks or months of natural daylight exposure (e.g., Voinchet et al., 2003; Jacobs et al., 2011; Demuro et al., 2015; Duval et al., 2017). Since incomplete signal resetting or residual subtraction can lead to non-trivial age overestimations, it is necessary to undertake site-specific assessments of bleaching sufficiency to ensure meaningful interpretations of resultant ESR or luminescence chronologies. For TT-OSL dating studies, singlegrain applications offer an ideal opportunity to better characterise TT-OSL signal resetting at the most fundamental scale of analysis (e.g., Arnold et al., 2019). Assessments of TT-OSL bleaching adequacy can also be undertaken via dating comparisons with more rapidly bleaching luminescence signals (e.g., post infrared infrared stimulated luminescence (pIR-IRSL and OSL signals); with parity in ages being used to support adequate resetting of the slower bleaching TT-OSL signal. In ESR dating studies, the multiple centre (MC) approach (Toyoda et al., 2000) has progressively become a standard requirement for assessing bleaching suitability prior to sediment deposition (see a recent overview in Duval et al., 2017). This comparative dating approach takes advantage of the differential bleaching rate of ESR signals associated with different lightsensitive paramagnetic centres in quartz (see bleaching kinetics comparison in Fig. 1 from Duval et al., 2017): 
namely, the slowly and incompletely bleached $\mathrm{Al}$ centre and the more rapidly (and fully) bleached Ti centres (Ti-Li and, if measurable, Ti-H centres). As part of the MC approach, various ESR signals are measured in a given quartz sample and, under ideal bleaching and transport conditions, they are expected to provide similar estimates of the dose absorbed by the sample during its burial history (e.g., see Sahnouni et al., 2018, and Voinchet et al., this volume, for examples of consistent Al and Ti ESR MC ages). Statistically significant differences in the resultant ESR chronologies of different paramagnetic centres are usually interpreted as indicating that the slowly bleaching Al centre provides a maximum age for the deposits, with the rapidly bleaching Ti centres most likely providing the closest estimate for the true age of sediment deposition (Toyoda et al., 2000; Tissoux et al., 2007; Voinchet et al., 2003; Duval et al., 2017).

The MC (Al and Ti) approach has recently been applied in a combined ESR-luminescence quartz dating study of the Porto Maior archaeological site, which is set in a fluvial terrace sequence of the Miño River (Galicia, northwest Spain; Méndez-Quintas et al., 2018). In that study, the ESR Al centre ages for the lower half of the fluvial sequence were found to be more than $50 \%$ older than the corresponding Ti-Li centre ages. The Ti centre ages at Porto Maior appear to be well-supported by paired post-infrared infrared stimulated luminescence (pIRIRSL) dating of potassium feldspar grains from closely associated samples (Méndez-Quintas et al., 2018), with both techniques converging on an age of $\sim 240 \mathrm{ka}$ for the main archaeological layer. This site provides an ideal opportunity for evaluating the usefulness of the ESR MC approach and for further investigating the age disparities obtained for the ESR Al and Ti centres by applying additional extended-range dating techniques such as single-grain TT-OSL. It also represents a suitable context for exploring the potential relationships between different quartz trapped charge dating signals (paramagnetic and luminescence), and for examining the potential and pitfalls of using these signals to resolve Middle Pleistocene human occupation histories at other archaeological sites in the region. These various issues are explored further in the present study.

Porto Maior represents the first reported Acheulean site in Europe to be characterised by an extensive accumulation of large cutting tools (LCTs). The density of LCTs at this site is higher than at any other western European site, and parallels that recorded at several sites from Africa and the Near East. Similarly, the large average size of the LCTs (predominantly handaxes) from Porto Maior, as well as their technological attributes, are comparable to those commonly found in Africa and the Near East (Méndez-Quintas et al., 2018). These shared lithic assemblage characteristics have been interpreted as representing evidence for a direct link between the Acheulean tradition from Africa and that of southwest Europe (Méndez-Quintas et al., 2018). Refining the accuracy and precision of existing age estimates at Porto Maior is therefore important for better understanding the chronological context of this important Lower Palaeolithic site within a regional archaeological framework.

Building on the recent archaeological evaluation of Porto Maior by Méndez-Quintas et al. (2018), the present study aims to undertake additional chronological assessments of the site using luminescence and ESR quartz dating comparisons. The specific aims of the paper are to: (i) expand the existing dating framework by obtaining new single-grain TT-OSL ages from selected Porto Maior samples and compare these with corresponding ESR Al and Ti quartz ages; (ii) derive a Bayesian modelled chronostratigraphic sequence for 
Porto Maior in order to derive combined ages for individual depositional units at the site, refine the timing of the in situ archaeological accumulation, and provide a stronger statistical foundation for future chronostratigraphic correlations across the basin; and (iii) examine the comparative optically bleached quartz dating results obtained from Porto Maior and a range of other published sites to better evaluate the empirical relationship between the bleaching properties and corresponding ages of ESR and TT-OSL signals in different sedimentary contexts.

\section{Study site, stratigraphy, lithic assemblage and existing chronology}

The archaeological site of Porto Maior is located along the northern margins of the Miño River (Galicia, Spain) on a fluvial terrace found $+34 \mathrm{~m}$ above the current river level (terrace $\mathrm{T} 4$ of the local fluvial terrace system). The $>6 \mathrm{~m}$ stratigraphic sequence is made up of five stratigraphic levels, characterised from bottom to top as follows: levels PM1 and PM2 - fluvial facies composed of clast-supported gravels; levels PM3 and PM4 fluvial facies composed of massive fine-grain (silts and sands) overbank deposits affected by pedogenesis, and; level PM5 - comprises a colluvium gravel deposit at its base (with re-deposited lithic industry) and fine loam sediments of aeolian origin in the upper section (Figure 1). The in situ anthropological accumulation of lithic tools at Porto Maior has been recovered from an $11.8 \mathrm{~m}^{2}$ excavated area at the base of PM4, which sits uncomfortably on PM3. In total, 290 lithic artefacts have been recovered from this fine-grain layer, of which 159 show no evidence of fluvial abrasion and are considered to represent localised human activity (denoted the main assemblage or MA in Méndez-Quintas et al., 2018). The LCT lithic assemblage has been described as large flake blank (LFB sensu Sharon) Acheulean and is dominated by handaxes, although it also contains cleavers and picks. The LCTs are among the largest reported for the Iberian Peninsula and Europe (mean length of handaxes $=186.4 \mathrm{~cm}$ ). and constitute the densest Acheulean assemblage published thus far for an European site $\left(9.5\right.$ pieces per $\left.\mathrm{m}^{2}\right)$. As noted in the Introduction section, both the technological attributes (including size) of the LCTs and accumulation patterns of the Porto Maior assemblage closely match those usually described for African and Near Eastern sites, potentially indicating an African affinity in the Acheulean tradition of southwest Europe. There are no preserved organic or faunal remains at Porto Maior (a common occurrence for open-air lower Palaeolithic sites in the region; e.g. Méndez-Quintas et al., in press), which precludes biochronological assessments of the archaeological sequence.

The existing chronology for the Porto Maior sedimentary sequence has been determined using a combination of three semi-independent quartz dating methods (Table 1): ESR dating of six quartz samples from units PM1, PM3, PM4 and PM5; potassium feldspar pIR-IRSL dating of four samples from units PM3, PM4 and PM5 using a stimulation temperature of $225^{\circ} \mathrm{C}$ and a preheat of $250^{\circ} \mathrm{C}$ for $60 \mathrm{~s}$ (herein denoted pIR-IRSL 225); and replicate single-grain OSL quartz dating of the luminescence sample collected from unit PM5 (Méndez-Quintas et al., 2018). The MC approach has previously been applied to each of the ESR quartz samples, resulting in two replicate ages per sample using the $\mathrm{Al}$ and Ti-Li (option D; Duval and Guilarte, 2015) centres. The suitability of the Ti-H signal has been additionally evaluated for the Porto Maior ESR samples by Méndez-Quintas et al. (2018). The five samples from the fluvial units (PM1, PM3 and PM4) did not yield sufficiently reliable Ti-H data to enable calculation of meaningful burial dose estimates. However, the uppermost sample collected from 
the colluvial and aeolian deposits of unit PM5 did produce a measurable Ti-H signal, and the resultant age was considered in the final dating evaluations of Méndez-Quintas et al. (2018), albeit with caveats (see details in section 3.2).

For the lower half of the sedimentary sequence (i.e., levels PM1 and PM3), the ESR ages from the Al centre were significantly (i.e., 58-70\%) older than those obtained for the Ti-Li centre. However, for the upper part of the sequence (i.e., levels PM4 and PM5), the Al and Ti-Li age estimates were in agreement at $2 \sigma$ (Table 1). In general, Méndez-Quintas et al. (2018) interpreted the ESR ages obtained using the Al centre as maximum (terminus post quem) ages due to the slow and incomplete bleaching characteristics of the Al signal (Duval et al., 2017), and considered the chronology derived using the more rapidly and completely bleachable Ti-Li centre as the most accurate estimate for the timing of sediment deposition. This assessment was confirmed by the pIR-IRSL 225 ages obtained for levels PM3 and PM4 (231 $\pm 15 \mathrm{ka}$ to $268 \pm 24 \mathrm{ka})$, which were in closer agreement with corresponding ESR ages obtained for the Ti-Li centre (206 $\pm 20 \mathrm{ka}$ to $279 \pm 26 \mathrm{ka})$ than those from the Al centre $(181 \pm 25 \mathrm{ka}$ to $451 \pm 66 \mathrm{ka})$. The only ESR age available for level PM1 $(264 \pm 27 \mathrm{ka}$, based on the Li-Ti centre) indicates that the basal unit of the sediment sequence was likely deposited during marine isotope stage (MIS) 8. Using the various ESR Ti-Li and pIR-IRSL ages, Méndez-Quintas et al. (2018) calculated weighted mean ages of $268 \pm 12 \mathrm{ka}$ and $226 \pm 10 \mathrm{ka}$ for units PM3 and PM4, respectively. The uppermost unit (PM5) was dated to MIS 2 using both single-grain OSL and pIR-IRSL. In contrast, the ESR Ti$\mathrm{H}$, Ti-Li and Al centres yielded systematically older ages of between $48 \pm 10 \mathrm{ka}$ and $138 \pm 25 \mathrm{ka}$ for related deposits in the same unit (Table 1), which were interpreted as maximum age estimates for PM5. The difference between the ESR and luminescence chronologies for this unit were considered to primarily reflect the limitations of applying ESR quartz dating over low natural dose ranges ( $<100 \mathrm{~Gy}$ ) when using the multiple aliquot additive dose method; although minor differences in the ESR and luminescence sampling positions within unit PM5 may have also contributed to these age inconsistencies. The difference in the combined age estimates of units PM4 and PM5 indicates the presence of a prolonged sedimentary hiatus at Porto Maior during the Late Pleistocene, which is consistent with a major change in sedimentary processes observed between levels PM4 and PM5 (Méndez-Quintas et al., 2018). The existing age range for the human occupation layer at the base of level PM4 is 293-205 ka, based on the $2 \sigma$ confidence interval of the weighted mean Ti centre and pIR-IRSL ages for units PM3-PM4.

\section{Methods}

\subsection{TT-OSL dating of sedimentary quartz grains from Porto Maior}

As part of an expanded quartz dating study of Porto Maior, we have applied single-grain TT-OSL to all four of the luminescence dating samples (PM16-3, PM16-4, PM16-5 and PM16-6) that were previously dated using pIR-IRSL 225 (Méndez-Quintas et al., 2018). Samples PM16-3 and PM16-5 come from the lower and upper part of unit PM3, respectively, and are closely associated with ESR samples VI1205 and VI1204. Samples PM16-6 and PM16-4 come from units PM4 and PM5, respectively, and are associated with ESR samples MIN1402 and MIN1401 (Table 1). Measurements of quartz TT-OSL signals were made using a Ris $\varnothing$ TL/OSL-DA-20 reader equipped with a spatially calibrated ${ }^{90} \mathrm{Sr} /{ }^{90} \mathrm{Y} \beta$ radiation source delivering a mean dose rate of $\sim 0.106$ Gy/s. The 
TT-OSL signal of individual grains were stimulated using a $10 \mathrm{~mW} \mathrm{Nd:YVO4} \mathrm{laser} \mathrm{attachment}(532 \mathrm{~nm}$, maximum power of $\sim 50 \mathrm{~W} \mathrm{~cm}^{2}$ ) at $90 \%$ power. Measurements were performed on $90-125 \mu \mathrm{m}$ quartz grains carefully brushed onto aluminium discs drilled with an array of $300 \times 300 \mu \mathrm{m}$ holes. This type of 'pseudo' single-grain approach was chosen because the proportion of grains yielding TT-OSL signals is relatively low for these samples (74-81\% of grain-hole positions did not produce any statistically distinguishable TT-OSL $\mathrm{T}_{\mathrm{n}}$ signal; Table S2). It is estimated that $\sim 18$ grains were placed in each hole position when using this configuration (Arnold et al., 2012a), but the low frequency of TT-OSL-producing grains for these samples (see section 4.1) effectively ensures single-grain resolution has been maintained in this study (Demuro et al., 2013). Ultraviolet emissions were detected using an EMI 9235QB photomultiplier fitted with a 7.5 mm-thick U-340 filter.

TT-OSL $\mathrm{D}_{\mathrm{e}}$ values were obtained using the single-aliquot regenerative-dose (SAR) protocol of Stevens et al. (2009), which has been modified to enable the measurement of individual grains (i.e., to enable single-grain measurements in steps 3, 5, 9 and 11 of Table 2; Demuro et al., 2014). Net $\mathrm{L}_{\mathrm{x}}$ and $\mathrm{T}_{\mathrm{x}}$ values were calculated by integrating the first $0.15 \mathrm{~s}$ of the TT-OSL decay curves and subtracting a background count from the last $0.25 \mathrm{~s}$ of the signal. The SAR dose-response curves were fitted using a single-saturating exponential function. Individual $D_{e}$ values were accepted if they passed the following quality assurance criteria: (i) the luminescence signals were statistically distinguishable from background (the net intensity of the natural test dose signal, $\mathrm{T}_{\mathrm{n}}$, was $>3 \sigma$ above the late-light background signal); (ii) the recycling ratio (i.e., sensitivity-corrected luminescence responses $\left(\mathrm{L}_{\mathrm{x}} / \mathrm{T}_{\mathrm{x}}\right)$ for two identical high regenerative doses) was consistent with unity at $2 \sigma$; (iii) the recuperation ratio, calculated as the ratio of the sensitivity-corrected $0 \mathrm{~Gy}$ dose point $\left(\mathrm{L}_{0} / \mathrm{T}_{\mathrm{x}}\right)$ to the sensitivitycorrected natural $\left(\mathrm{L}_{\mathrm{n}} / \mathrm{T}_{\mathrm{n}}\right)$, was $<5 \%$; (iv) no contamination by feldspar grains or inclusions was detected using the OSL IR depletion ratio (i.e., the ratio of the $\mathrm{L}_{\mathrm{x}} / \mathrm{T}_{\mathrm{x}}$ values obtained for two identical regenerative doses measured with and without prior IR stimulation overlapped with unity at $2 \sigma$; Duller, 2003); (v) the net $\mathrm{T}_{\mathrm{n}}$ signal had a relative error of $<30 \%$; (vi) the sensitivity-corrected natural signal intercepted the sensitivity-corrected dose-response curve and it did not intercept the saturated part of the dose-response curve (i.e., the $L_{n} / T_{n}$ value was lower than the $I_{\max }$ saturation limit of the dose-response curve at $2 \sigma$ ); (vii) the dose-response curve did not display anomalous properties (e.g., zero or negative responses with increasing dose) and resulted in good Monte Carlo fits.

Dose recovery tests were performed on sample PM16-3 after administering a dose of $578 \pm 4$ Gy on top of the naturally accumulated dose for a sub-set of grains (see Demuro et al., 2019 for full details of procedure). A net dose recovery (measured-to-given dose) ratio of $0.98 \pm 0.26$ was obtained after subtracting the weighted mean natural $D_{e}$ of sample PM16-3 (Table 3) from the weighted mean $D_{e}$ of the unbleached and dosed grains, and dividing this value by the given dose (Table S1). Although this dose recovery result is based on a limited number of grains $(n=18)$, the ratio obtained is within $2 \sigma$ of unity and supports the suitability of the chosen measurement conditions for dating these samples. 
The environmental dose rates used to calculate the final single-grain TT-OSL ages of samples PM16-4, PM166, PM16-5 and PM16-3 have been obtained using a combination of in situ and laboratory measurements (Table 3). Gamma dose rates have been derived from in situ gamma-ray spectrometry measured immediately after collecting the luminescence dating samples (using the windows approach; Arnold et al., 2012b). Additionally, sediment sub-samples were collected from each sample position to assess sample moisture content and beta dose rate values. The water content values (expressed as percentage of dry weight) were set to between $20 \%$ and 29\% for samples PM16-5, PM16-3 and PM16-6 (from levels PM3 and PM4) and 15\% for sample PM16-4 (from the uppermost level PM5). These water content values were equivalent to those used for calculating pIRIR and ESR ages reported in Méndez-Quintas et al. (2018). Beta dose rates were determined from low-level beta counting measurements undertaken on dry and homogenised sediment samples (Bøtter-Jensen and Mejdahl, 1988). The contribution from cosmic rays was calculated using the approach outlined in Prescott and Hutton (1994). Full details of the dose rate measurement conditions and calculations can be found in the footnotes of Table 3 .

\subsection{Bayesian age modelling methodology}

Bayesian age modelling has been used to combine the reliable luminescence and ESR dating results (likelihoods) from the present study and Méndez-Quintas et al. (2018) with all known stratigraphic information (priors) for Porto Maior, in order to derive a unified chronostratigraphic framework. The specific aims of the Porto Maior Bayesian modelling assessments are threefold: (i) to calculate amalgamated (posterior) probabilistic chronologies for individual stratigraphic units; (ii) to improve the existing age estimate for the in situ archaeology-bearing deposits, which is currently based on the weighted mean age estimate of bracketing Ti centre and pIR-IRSL ages from units PM3-PM4, and; (iii) to undertake sensitivity analyses of different types of dating uncertainty representation within OSL and ESR chronological modelling frameworks. The Porto Maior Bayesian age model has been constructed using OxCal v4.3.2 (Bronk-Ramsey, 2009a), and is based on the approach outlined in Hamm et al. (2016) and Demuro et al. (2019). A sequence deposition model with nested sub-sequences of uniform prior duration and associated boundaries has been used to capture the chronostratigraphic features of the site. The main depositional sequence incorporates four stratigraphic units in an ordered succession according to depth (units PM1-2, PM3, PM4 and PM5, from the base of the sequence upwards). Units PM1 and PM2 are represented as a combined unit in the model as they are sedimentologically similar, comprise archaeologically sterile fluvial facies, and they are collectively constrained by only a single likelihood age estimate (ESR sample MIN1403 from PM1). Individual stratigraphic units have been represented as nested sequences within the broader depositional framework since their overall stratigraphic ordering is sufficiently well-preserved. The relative ordering of dating samples from individual units is also sufficiently clear and therefore all likelihood groupings have been modelled as single sequences rather than sub-phases. Boundaries have been used to delineate the beginning and end of each stratigraphic unit. The entire site sequence has been constrained with a minimum age of 0 years for the uppermost boundary (above the PM5 sequence) and a maximum age of 424 ka for the lowermost boundary (below the PM1-2 sequence). The latter is based on the ESR Al-centre age for sample MIN1403, which provides a maximum age estimate for the deposition of unit PM1 according to the MC ESR dating approach (Méndez-Quintas et al., 2018). 
Fifteen numerical dating results obtained using five different luminescence and ESR signals (ESR Ti-Li centre, ESR Ti-H centre, single-grain OSL, single-grain TT-OSL and pIR-IRSL) and two different preserved minerals (quartz and potassium feldspar extracts) have been included as likelihoods in the Porto Maior Bayesian model (Table 4). The six ESR likelihoods included in the model have been derived using the most rapidly bleached Ti centre signal measured for each dating sample (Ti-Li in the case of samples MIN1402, MIN1403, V1204, V1205, V1206 and Ti-H for MIN1401; see Méndez-Quintas et al., 2018 for full details). The more slowly (and incompletely) bleached $\mathrm{Al}$ centre signals are considered to yield terminus post quem ages according to ESR dating MC approach employed by Méndez-Quintas et al. (2018), and have therefore not been included as likelihoods in the Bayesian model to ensure quality control. For sample MIN1401, Méndez-Quintas et al. (2018) consider the Ti-H age to be more appropriate than the replicate Ti-Li or Al centre ages but caution that the reliability of the Ti-H likelihood may be compromised by the proximity of the natural $\mathrm{D}_{\mathrm{e}}$ value to the practical lower dating limit of the ESR technique and the poor goodness-of-fit of the extrapolated multiple aliquot additive dose response curve. These complications have been accounted for in our model by assigning sample MIN1401 as a maximum age likelihood for the lowermost (bottom) boundary of the PM-5 sequence using the OxCal after command.

The replicate pIR-IRSL and TT-OSL dating results for samples PM16-3, PM16-5 and PM16-6, and the replicate pIR-IRSL, OSL and TT-OSL dating results for sample PM16-4 are considered equally reliable on methodological grounds (see section 4.1 and discussions in Méndez-Quintas et al., 2018), and have therefore been included in the Bayesian model as combined luminescence age estimates for each sample. Since the replicate age determinations for these samples do not have fully independent dose rate uncertainties, they were combined after excluding all shared systematic uncertainty terms (including beta source calibration uncertainty, long-term water content uncertainty, cosmic-ray and internal dose rate errors, and uncertainties on dose rate conversion factors). This was achieved in OxCal by inputting the TT-OSL $D_{e}$ estimate, pIR-IRSL $D_{e}$ estimate, OSL $\mathrm{D}_{\mathrm{e}}$ estimate and shared dose rate term as individual parameters with associated random uncertainty terms. The pIR-IRSL+TT-OSL and pIR-IRSL+OSL+TT-OSL ages of individual samples were then calculated as dependent parameters, and a weighted average was derived using the OxCal combine function. The additional component of shared systematic uncertainty was subsequently added in quadrature to the combined age for samples PM16-3, PM16-4, PM16-5 and PM16-6 for the purpose of Bayesian modelling.

The luminescence and ESR likelihoods were input into the model as calendar ages before AD2014 (the average year of sample collection), together with their associated $1 \sigma$ uncertainty ranges, using the date command. Individual likelihoods have been represented in the main Bayesian model (denoted hereafter as "Model 1") using their combined systematic and random uncertainty terms, following the approach adopted in similar studies of multi-method chronological datasets (e.g., Falguères et al., 2013; Higham et al., 2014; Kennett et al., 2015; Hamm et al., 2016; Demuro et al., 2019). While this approach may be considered sub-optimal for certain Bayesian age modelling applications (e.g., Rhodes et al., 2003), alternative modelling of shared systematic uncertainties in Bayesian frameworks such as OxCal can be limited by practical considerations (e.g., 
subsequent recombination of systematic uncertainty components with modelled age distributions may be complicated by non-Gaussian posterior distributions). For the type of prior and likelihood datasets considered in the present study, it has also been reported that Bayesian age modelling of ex ante combined systematic and random uncertainty terms may not significant bias posterior age estimates (e.g., Higham et al., 2014; Groucutt et al., 2018; Demuro et al., 2019). However, in order to better assess the sensitivity of the Porto Maior Bayesian modelling results to prior incorporation of the shared and systematic dating uncertainties, we have implemented a second version of the model ("Model 2') in which only the random luminescence and ESR dating uncertainty ranges (i.e., those shown in parentheses in Table 1) have been included as likelihood input parameters. This modelling approach follows the procedure outlined in Clarkson et al. (2017), and involves subsequently recombining the average relative systematic uncertainty of the fifteen likelihood estimates with the modelled posterior boundary age distributions of each stratigraphic unit ex post facto. For the fifteen numerical dating estimates included in our model, the mean random and systematic uncertainty components account for $8.1 \pm$ $1.1 \%$ and $6.3 \pm 0.3 \%$ (mean \pm standard error), respectively, of the total (random plus systematic) relative $1 \sigma$ uncertainties before combining in quadrature. The total (random plus systematic) uncertainties associated with the modelled upper and lower age ranges of each unit (top and bottom boundary posterior distributions) have therefore been calculated by combining, in quadrature, the modelled (random) uncertainties and the 6.3\% mean relative systematic uncertainty derived from the original likelihood dataset.

The two versions of the Porto Maior Bayesian age model were run using the general outlier function (Bronk Ramsey, 2009b), which is based on a Student t-test distribution with 5 degrees of freedom. Prior outlier probabilities of $5 \%$ were equally assigned to all dating samples to identify potentially significant statistical outliers. Likelihood estimates that yielded posterior outlier probabilities $>5 \%$ were not excluded from the final model but were proportionally down-weighted in the iterative Monte Carlo runs, thereby producing an averaged chronological model (Bronk Ramsey, 2009b).

The OxCal date and difference functions (Bronk Ramsey, 2009a) were used to calculate the duration of each stratigraphic unit and potential hiatuses between sedimentary deposits from the posterior probabilities of their associated upper and lower boundaries. For Model 2, the difference and date queries have been run on the posterior boundary distributions after recombining, in quadrature, the mean relative systematic uncertainty of the thirteen likelihood estimates with the modelled (random) uncertainty ranges.

\section{Results}

\subsection{Single-grain TT-OSL characteristics and ages}

Relatively few of the quartz grains measured for samples PM16-4, PM16-6, PM16-5 and PM16-3 yield useable TT-OSL signals; 87-92\% of grain-hole positions ('pseudo' single-grain measurements) exhibit absent or very weak TT-OSL signals (failing quality assurance criteria (i) and (v)), and only $\sim 1-2 \%$ of measured $\mathrm{D}_{\mathrm{e}}$ values are considered suitable for burial dose estimation (Table S2). Low-to-moderate sensitivity change in the TT-OSL signal was observed through the SAR measurement procedure, with more than half of the grains accepted for final age estimation displaying sensitivity changes (i.e., an increase in $\mathrm{T}_{\mathrm{x}}$ signal relative to $\mathrm{T}_{\mathrm{n}}$ ) of less than $50 \%$. 
The accepted grains from these samples generally exhibit fast-decaying TT-OSL signals, consistent with charge transfer into the most readily bleached (so-called 'fast') OSL dating trap, and the dose-response curves display high saturation dose limits $\left(\mathrm{D}_{0}\right.$ values $>500 \mathrm{~Gy}$; i.e., sufficient to accommodate the expected burial doses range of 700-900 Gy for these samples according to the associated pIR-IRSL ages) (Figure 2a and b).

The single-grain TT-OSL $D_{e}$ distributions display a moderate degree of scatter (Figure 2c-f), although the relative uncertainties of the individual $D_{e}$ values are generally high (20-60\%) due to the dim TT-OSL signal intensities of accepted grains. None of the $\mathrm{D}_{\mathrm{e}}$ datasets are considered to be significantly positively skewed according to the criterion outlined by Arnold and Roberts (2011). The overdispersion values obtained for samples PM16-4, PM16-6, PM16-5 and PM16-3 range between 32\% and 47\% (Table 3). These values are higher than those reported elsewhere for well-bleached and unmixed single-grain $\mathrm{D}_{\mathrm{e}}$ datasets (e.g., the metaanalysis overdispersion averages of $20 \pm 1 \%$ and $21 \pm 2 \%$ reported for single-grain OSL and single-grain TTOSL datasets by Arnold and Roberts, 2009 and Arnold et al., 2019, respectively). However, they are in agreement with these published average overdispersion ranges when compared at $2 \sigma$. The above-average overdispersion values for the Porto Maior samples may be related to intrinsic 'between-grain' scatter associated with our experimental procedures (e.g., grain-to-grain variations in luminescence responses due to the fixed SAR conditions; e.g., Demuro et al., 2013) or microdosimetry effects (e.g., Nathan et al., 2003; Mayya et al., 2006; Arnold et al., 2014) arising from variable proximities of quartz grains to either quartzite pebbles with relatively low radioactivities or potassium feldspars grains with relatively high radioactivities. Taking into consideration the stratigraphic context and $\mathrm{D}_{\mathrm{e}}$ characteristics of the Porto Maior samples, we have derived representative single-grain TT-OSL burial dose estimates using the weighted mean $\mathrm{D}_{\mathrm{e}}$ values for each sample, which has been calculated using the central age model (CAM) of Galbraith et al. (1999). The final quartz TTOSL ages for the lower (sample PM16-3) and upper (sample PM16-5) sections of unit PM3 are $242 \pm 32 \mathrm{ka}$ and $225 \pm 31 \mathrm{ka}$, respectively (Table 3), which are consistent with the existing pIR-IRSL ages for this unit at $1 \sigma$ (Table 1). For level PM4 (sample PM16-6) the final single-grain TT-OSL ages are $172 \pm 21 \mathrm{ka}$ and this age is within $2 \sigma$ of the equivalent pIR-IRSL age for this unit. For level PM 5 (sample PM16-4) the final TT-OSL age is $26 \pm 3 \mathrm{ka}$, which is within $2 \sigma$ the single-grain OSL age obtained for this sample, but slightly older than the corresponding pIR-IRSL age at $2 \sigma$ (Table 1).

\subsection{Bayesian modelling results}

The results of the Porto Maior Bayesian model (Model 1) are summarised in Table 4 and Figure 3. All modelled age ranges have been rounded to the nearest 50 years and are reported as the $68.2 \%$ and $95.4 \%$ highest probability density function (PDF) ranges, and the mean and $1 \sigma$ uncertainty ranges of the modelled posterior distributions. The Bayesian modelled posterior age ranges of the upper and lower (top and bottom) boundaries were used to constrain the end and beginning periods of each depositional event at Porto Maior, as well as the likely age and duration of each stratigraphic unit and potential hiatuses between sedimentary deposits. These chronological relationships are summarised in Figure $\mathbf{4}$ and Table 5, and have been calculated using the difference and date query functions in $\mathrm{OxCal}$ (see Table 5 footnote for further details). 
The statistical validity of Model 1 is supported by a model agreement index $\left(A_{\text {model }}\right)$ of $128.8 \%$, which is considerably higher than the preferable internal consistency threshold value of 60\% (Bronk-Ramsey, 2009a), and indicates significant overlap between the measurement data and the modelled posterior distributions as a whole. The overall agreement index $\left(A_{\text {overall }}\right)$ of Model 1 is $126 \%$, which reflects relatively high individual agreement indices $\left(A_{i}=\right.$ correspondence between individual likelihood and posterior distributions) for each modelled dating sample, as well as a strong convergence integral (an assessment of whether the modelling algorithm has generated a truly representative posterior probability distribution) (Bronk-Ramsey, 2009a). The individual $A_{i}$ values were also significantly higher than $60 \%$ for all modelled likelihoods, ranging from $78.4 \%$ for ESR MIN1403 to $128.6 \%$ for pIR+TT-OSL PM16-5 with a likelihood average $A_{i}$ of $107.5 \%$. The average convergence integral for all individual posterior distributions is $96.4 \%\left(C_{i}\right.$ range $\left.=85.6-100 \%\right)$, supporting the overall effectiveness of the Monte Carlo solutions. Sample ESR VI1204 was the only likelihood to exhibit a posterior outlier probability higher than the specified prior threshold of 5\% (Table 4); though this occurrence is not considered extraordinary as one in twenty individual age estimates are expected to be significantly offset from the event of interest when adopting a prior outlier threshold of 5\% (Bronk Ramsey, 2009b). This sample also exhibits an acceptable $A_{i}$ value of $96.8 \%$ suggesting that it does not constitute a major statistical outlier. The overall suitability of the modelling indices and absence of major statistical outliers supports the appropriateness of the Bayesian modelling results for deriving meaningful combined age estimates for individual depositional units at Porto Maior.

The modelled posterior distributions of all the numerical dating samples (shown in dark blue on Figure 3) encompass narrower age ranges in comparison to their original likelihood age ranges (shown in light blue on Figure 3). Overall, the relative uncertainty ranges of the posterior likelihood distributions are reduced by an average of $32 \%$ when compared with the original dating sample uncertainties. The improvement in precision is most apparent for the four posterior likelihood estimates from unit PM3 (pIR+TT-OSL PM16-5, ESR VI1204, ESR VI1205, pIR+TT-OSL PM16-3), which exhibit 47-55\% reductions when compared with the original dating sample uncertainties. For the fifteen dating samples included in our model, the random and systematic uncertainty components account for $8.1 \pm 1.2 \%$ and $2.6 \pm 0.3 \%$ (mean \pm standard error), respectively, of the final (random plus systematic) relative $1 \sigma$ uncertainties (mean $=10.6 \pm 0.9 \%$ ) after combining in quadrature. The modelled posterior age distributions of these samples exhibit optimised total relative uncertainties of 4.2$20.0 \%$ (mean $=6.6 \pm 0.5 \%$ ) (Table 4), which remain significantly higher than the relative systematic uncertainty contributions to the original likelihood ranges $(1.1-4.7 \%$; mean $=2.6 \pm 0.3 \%)$. As the overall improvements in chronological precision for Model 1 do not exceed the underlying systematic uncertainty contributions to the likelihood estimates, it seems unlikely that our modelling approach will have significantly biased the posterior age estimates. This interpretation is further supported by the results of Model 2, as detailed below.

The results of Porto Maior Bayesian Model 2, which was run after excluding all shared systematic uncertainty components from each luminescence and ESR likelihood input parameter, are summarised in Table S3-S4 and Figures S1-S2. The Model 2 posterior boundary distributions in Table S3 are expressed in two formats: the 
first (shown outside parentheses) represents the raw OxCal modelling output and is based on propagation of random dating uncertainties only, while the second (shown in parentheses) includes propagation of the modelled (random) dating uncertainties and the mean systematic uncertainty (6.3\%) of the fifteen numerical dating estimates in quadrature. The latter modelled age estimate is therefore the most appropriate to use for direct comparisons with independent chronologies and with the results of Model 1. Model 2 exhibits slightly lower $A_{\text {model }}$ and $A_{\text {overall }}$ indices (101.1 and 102.7, respectively) compared to Model 1, though both exceed the preferable acceptance threshold value of 60\% (Bronk-Ramsey, 2009) and are indicative of adequate modelling agreement. As with Model 1, none of the posterior likelihood estimates are identified as major statistical outliers and sample ESR VI1204 is again the only likelihood exhibiting a posterior outlier probability higher than the specified prior threshold of 5\% (Table S3).

After recombining the shared systematic uncertainties with the posterior age distributions of Model 2, the resultant boundary age ranges of each stratigraphic unit reveal relatively minor differences between the two modelling scenarios (Table 6). The Model 2 mean posterior age ranges for the upper and lower boundaries (top and bottom) of each stratigraphic unit differ from the corresponding estimates of Model 1 by -800 years on average (a mean difference of $-0.3 \%$ between the modelled boundary ages of Model 2 and 1 ). The total $1 \sigma$ relative uncertainties on the Model 2 mean boundary ages were on average 1\% higher than their Model 1 counterparts. Application of a paired-age homogeneity test (adapted from Galbraith, 2003; see Appendix A8 of Galbraith and Roberts, 2012) reveals that all of the corresponding mean boundary ages calculated using Model 1 and Model 2 are in agreement with a common value at their $2 \sigma$ uncertainty ranges (Table 6; see footnote for further methodological details). To further examine the consistency of the modelling results as a combined dataset, we have applied a one-sample two-tailed Student's $t$-test following the approach detailed in Arnold et al. (2015). Standardised normal values $(x)$ were first calculated as the difference between the Model 1 and Model 2 mean age of each boundary divided by the square root of the summed squares of the corresponding $1 \sigma$ age uncertainties. The observed value of the test statistic $\left(t_{\mathrm{obs}}\right)$ was then calculated from the standardised normal values of each dataset according to the equation:

$t_{o b s}=\frac{\left(\tilde{x}-\mu_{0}\right)}{\left(\frac{s}{\sqrt{n}}\right)}$

where $\tilde{x}$ is the sample mean standardised normal value, $\mu_{0}$ is the reference value (in this case 0 ), and $s$ is the standard deviation of the standardised normal values. The observed $t$ value $\left(t_{o b s}\right)$ was compared with critical test statistic value $\left(t_{\text {crit }}\right)$ at the 0.05 significance level with $n-1$ degree of freedom. The results of this statistical test reveal that the paired Model 1 - Model 2 boundary age estimates do not differ from each other at the 95.4\% confidence level $\left(t_{o b s}=0.26<t_{\text {crit }(\alpha: 0.05)}= \pm 2.36\right)$ (i.e., the null hypothesis is accepted).

In assessing the relative merits of Model 2 and Model 1 for deriving the final chronological sequence of Porto Maior it is worth noting that several of the modelled boundaries exhibit non-Gaussian posterior age distributions. This is particularly true for the upper and lower boundary of PM5, the lower boundary of PM4 
and the lower boundary of PM1-2 (Figures 3, S1). For such non-Gaussian posterior age distributions, the recombination of a simplified (effectively Gaussian) mean relative systematic uncertainty derived from the original likelihood dataset may not adequately represent or capture the true underlying complexity of the total modelled uncertainty ranges. Model 2 is also limited by the fact that it requires recombination of a single, generalised estimate of the likelihood relative systematic uncertainty (6.3\%) with the modelled (random) boundary age uncertainties ex post facto. This approach does not directly capture the inherent inter-sample variability in relative systematic uncertainties for the Porto Maior sequence, which vary between $4.9 \%$ and $7.7 \%$ for individual likelihood estimates (before combining in quadrature). Both of these practical complications are avoided in Model 1, as the final non-Gaussian posterior boundary distributions have been calculated by considering sample-specific systematic uncertainties and prior incorporation of all relevant empirical uncertainty components upfront. As such, we favour Model 1 for reconstructing the final chronostratigraphic sequence of Porto Maior. While acknowledging the assumptions and simplified likelihood representations implicit in the Bayesian approach of Model 1, it is worth emphasising that the resultant posterior boundary distributions are statistically indistinguishable from those obtained using the Model 2 configuration. The modelling results are therefore relatively insensitive to the treatment of shared likelihood uncertainties in this instance, and can be considered as a useful probabilistic means of evaluating age-depth relationships at the site.

\section{Discussion}

\subsection{Comparison between TT-OSL and ESR chronologies for Porto Maior}

This study (together with Méndez-Quintas et al., 2018) represents one of the few available comparisons of ages obtained using two ESR centres in combination with TT-OSL. The new dating results presented here therefore provide a useful means of assessing the empirical relationship between the ESR and TT-OSL signals for optically bleached quartz. ESR sample MIN1402, which was collected from the upper part of the Porto Maior sequence (level PM4), produced similar ages (within $1 \sigma$ errors) for the Al (181 $\pm 25 \mathrm{ka}$ ) and Ti-Li (option D; $206 \pm 20 \mathrm{ka}$ ) centres. The single-grain TT-OSL age obtained for the correlative luminescence sample in level PM4 was $172 \pm 21 \mathrm{ka}$, which is within $1 \sigma$ of both the Al and Ti-Li ages. In the lower section (levels PM3 and PM2), the ESR ages of samples VI1204, VI1205 and MIN1403 obtained using the Al centre were older by a factor of 1.6-1.8 ( 424-452 ka) when compared to the ages obtained with the Ti-Li centre ( 264-279 ka). The two TT-OSL ages of $225 \pm 31 \mathrm{ka}$ and $242 \pm 32 \mathrm{ka}$ presented here for the luminescence samples collected from level PM3 (PM16-3 and PM16-5, respectively), as well as the pIR-IRSL ages presented in Méndez-Quintas et al. (2018) for this level ( 259-268 ka), are in closer agreement with the ESR ages obtained with the Ti-Li centre than those obtained with the Al centre (Table 1). These results indicate a potentially weaker link between the ESR Al centre and the trap giving rise to the TT-OSL signal in quartz, when compared to the Ti-Li centre.

One aspect worth considering further when evaluating the comparative quartz dating results for Porto Maior is that the TT-OSL measurements have been made at the single-grain scale of analysis while the ESR measurements have been made on bulk aliquots containing between 50 and $250 \mathrm{mg}$ of quartz grains, and 
therefore reflect composite signals derived from >10,000 grains (see Duval et al., 2017). The ESR experimental set-up therefore precludes direct assessment of grains with potentially problematic ESR signals that could have affected the resulting age estimates. By definition, bulk ESR $D_{e}$ measurements are only likely to be reliable if the combined multi-grain signal is dominated by individual quartz grains with similar natural bleaching histories and radiation sensitivity behaviours. However, the proportion of grains that actually contribute to the main radiation-induced ESR signals measured from multigrain aliquots is presently unknown, and the information available regarding grain-to-grain ESR signal variability is scarce. Single-grain quartz ESR measurements made using Q-band spectroscopy (Beerten and Stesman, 2006) have showed that individual grains in a given sample can display significant variations in behaviours, suggesting that the ESR signal intensity measured on a given multigrain aliquot may potentially be composed of grains that contribute differently to the bulk signal. However, as with single-grain quartz OSL characteristics, it is feasible that different samples may exhibit significant variability in their grain-to-grain ESR signal properties and relative signal contributions. Unfortunately, limitations in the standard instrumentation currently available for ESR dating mean it is virtually impossible to evaluate possible grain averaging effects on our ESR $\mathrm{D}_{\mathrm{e}}$ evaluations for the Porto Maior samples. It therefore remains difficult to ascertain a direct correspondence between TT-OSL and ESR signal properties at the individual grain scale in this study.

Although this study does not directly investigate the causes behind the closer age agreement between the TTOSL and Ti-Li signals when compared to the Al signal, one possible explanation for this observation may relate to partial bleaching of the Al centre for the lower deposits. This possibility would be consistent with the recent study of Duval et al. (2017), which directly measured the bleaching rates of the ESR Al, Ti-Li and Ti-H centres, as well as TT-OSL signals, for a range of Spanish and French sedimentary quartz samples. The bleaching rate of the various ESR signals and the TT-OSL signal was measured after exposing aliquots of naturally-irradiated quartz to UV light in a UVACUBE 400 (Dr Honle) sunlight simulator for different time-periods. The results showed that the TT-OSL signal was optically reset at a rate similar to the Ti-H and Ti-Li centres combined, while the bleaching rate of the Al centre was substantially slower when compared to all other ESR signals and the TT-OSL signals. It is worth noting, however, that the results presented in Duval et al. (2017) relate to ESR and TT-OSL measurements made on different samples, and therefore they may mask inter-sample variations in bleaching properties. Several other ESR quartz studies have shown that the Al centre bleaches more slowly than any of the Ti centres when exposed to UV light and that an unbleachable component is prevalent in the $\mathrm{Al}$ centre signal (Walther and Zilles, 1994; Toyoda et al., 2000; Voinchet et al., 2003; Tissoux et al., 2007, 2008). Gao et al. (2009) showed that the ESR Ti-Li centre (option A sensu Duval and Guilarte, 2015) signal is readily bleached by UV exposure and that signal depletion occurs more rapidly as the UV intensity is increased. Although equivalent data is not available for the TT-OSL signal, sunlight bleaching experiments performed in different natural environments are consistent with similar dependencies on UV light intensity. In particular, comparison of the results presented by Demuro et al. (2015) and Jacobs et al. (2011) may indicate that the TTOSL signal bleaches twice as fast at higher altitudes (950 m.a.s.l), where the UV radiance is considered to be more intense, than at sea level (i.e., $90 \%$ of the original TT-OSL signal was depleted by 6 weeks in the former study versus 12 weeks in the latter study). Again, it would be worth replicating these UV dependency tests 
using the same samples and the same experimental set-up to eliminate any sample-specific or empirical biases. Nevertheless, the comparative ESR and luminescence datasets obtained for the two Porto Maior samples are outwardly consist with existing research on the relative bleaching characteristics of the Al centre, Ti-Li centre, Ti-H centre and the TT-OSL signals of quartz, which also suggest a closer relationship between the trap giving rise to the TT-OSL signal and the ESR Ti centres, rather than the Al centre.

\subsection{Comparison of published TT-OSL and ESR chronologies for different sites}

To better evaluate the relationship between ESR and single-grain TT-OSL dating signals across a broader range of sites and sedimentary contexts, we have synthesised the comparative ages published so far as part of combined ESR-TT-OSL dating studies (Table 7, Figure 5). In total, ESR and single-grain TT-OSL dating have been applied in conjunction at eight archaeological and palaeoenvironmental sites, with most of these comparative studies focusing on sedimentary sequences from the Iberian Peninsula. The MC ESR dating approach has been employed at five of these study sites, with the remaining sites being ESR dated using $\mathrm{Al}$ centre signals alone (Table 7). Arnold et al. (2013) reported single-grain and multigrain TT-OSL ages of 352 and $\sim 32 \mathrm{ka}$, respectively, for the formation of the $+19-30 \mathrm{~m}$ terrace of the Pico River (Duero basin, Spain). These ages were in agreement with ESR Al centre ages of $\sim 370$ and $\sim 400$ ka derived from a correlative terrace in the neighbouring Arlanzon River valley (terrace Tazn8; Moreno et al., 2012). There has been less success, however, in obtaining reproducible ESR Al centre and single-grain TT-OSL ages at the nearby karstic infill deposits of Atapuerca, which are located adjacent to the Arlanzon River ( $10 \mathrm{~km}$ away). In the combined single-grain TT-OSL and ESR dating study of the Middle Pleistocene hominin site of Sima de los Huesos, two of the three dating samples collected from stratigraphic layer LU-7 produced ESR Al centre ages that were systematically older than corresponding single-grain TT-OSL ages by a factor of two or three (i.e., $\sim 1.5 \mathrm{Ma}$ and 945 ka compared to a weighted mean TT-OSL age of $416 \pm 19 \mathrm{ka}$ ). These two Al centre ages were also significantly older than U-series ages obtained on calcite rafts $(436 \pm 30 \mathrm{ka} ; \mathrm{n}=4)$ from the underlying stratigraphic layer (LU-6) (Arsuaga et al., 2014). This systematic age overestimation relative to several independent dating methods (U-series, single-grain TT-OSL, pIR-IR ${ }_{225}$ ) may be partially explained by the deep karst setting of the site and the potentially reduced optical bleaching possibilities of the Al centre signal in this depositional setting when compared to open-air sites (i.e., fluvial deposits). Interestingly, no evidence of partial bleaching has been observed in the single-grain TT-OSL $D_{e}$ datasets from Sima de los Huesos (Arnold et al., 2014; Demuro et al., 2019), further supporting our observations at Porto Maior that there seems to be a weak link between the ESR Al centre and the trap giving rise to the single-grain TT-OSL signal. Elsewhere in the Atapuerca complex, Moreno et al. (2015) obtained three ESR Al centre ages of $591 \pm 71 \mathrm{ka}$ to $947 \pm 90 \mathrm{ka}$ for Gran Dolina level ATD6-3, which has been independently dated to >780 ka using palaeomagnetism (Parés et al., 2013), 780-949 ka using combined US-ESR dating on a Homo antecessor tooth (Duval et al., 2018), and 831-856 ka using single-grain TT-OSL dating (Arnold et al., 2015; Arnold and Demuro, 2015). The weighted mean ESR and TT-OSL ages for Gran Dolina level TD6-3 are in broad agreement at $2 \sigma$, though the three Al centre ages exhibit a significant amount of scatter that is likely related to the heterogeneous dosimetric environment and the lack of in situ dosimetry measurements for two of the samples. 
The ESR MC approach has been applied in conjunction with single-grain TT-OSL dating at a series of Early Pleistocene ( 1-1.4 Ma) fluvial deposits from Morocco, as well as Middle Pleistocene fluvial deposits from France and Spain (Table 7). For the Early Pleistocene fluvial sequences of the Moulouya River basin, Morocco, both the $\mathrm{Al}$ and Ti-Li centres produced ESR ages that are in agreement with the paired TT-OSL ages at $2 \sigma$; although the Ti-Li centre (option D) produced slightly younger ages that are in closer agreement with those obtained with single-grain TT-OSL (Bartz et al., 2018; 2019). Similar trends are apparent when comparing the combined Al centre, Ti centre and single-grain TT-OSL ages obtained for the Lower Palaeolithic fluvial sequences of Lunery-la Terre-des-Sablons and Brinay-la Noira in the Middle Loire basin, France (Duval et al., this volume). Finally, fluvial deposits corresponding to the formation of the $+20 \mathrm{~m}$ terrace in the Deba River system of Cantabria (northern Spain) have been dated to $216 \pm 15 \mathrm{ka}$ using single-grain TT-OSL (Arriolabengoa et al., 2018). Comparison of this TT-OSL age with the MC ESR results presented for the same terrace (del Val et al., 2019), reveals close agreement with the both Ti-Li centre option D and Ti-H centre ages of $259 \pm 20 \mathrm{ka}$ and $192 \pm 21 \mathrm{ka}$. In contrast, the corresponding Al centre age of this terrace is $421 \pm 27 \mathrm{ka}$ (del Val et al., 2019), which is older than the TT-OSL age by a factor of two.

Collectively, the published datasets synthesised in Figure 5 and Table 7 indicate that the comparative Ti-Li centre (option D) and single-grain TT-OSL ages obtained so far on optically bleached quartz are in agreement at $2 \sigma$. Though based on a limited sample dataset $(n=9)$, the combined Ti-Li centre - single-grain TT-OSL dataset is well-represented by a weighted linear regression function $\left(\mathrm{R}^{2}=0.942\right)$ that is consistent with the $1: 1$ line at $2 \sigma($ slope $=1.15 \pm 0.08$; intercept $=101 \pm 20 \mathrm{ka})($ Figure 5a). Application of a paired-age homogeneity test (adapted from Galbraith, 2003; see Appendix A8 of Galbraith and Roberts, 2012) reveals that 89\% of the samples have $\mathrm{Ti}$ centre and single-grain TT-OSL ages in agreement with a common value at their $2 \sigma$ uncertainty ranges; while $33 \%$ of these samples yielded paired ages that are consistent with a common value at their $1 \sigma$ uncertainty ranges. To assess the general consistency of the Ti-Li centre and single-grain TT-OSL age estimates as a single dataset, we applied the one-sample two-tailed Student's $t$-test outlined in Section 4.2, following the approach detailed in Arnold et al. (2015). The results of this statistical test reveal that the paired Ti-Li centre - single-grain TT-OSL ages estimates do not differ from each other at the $95.4 \%$ confidence level $\left(t_{o b s}=1.82<t_{\text {crit }(\alpha: 0.05)}= \pm 2.36\right)$ (i.e., the null hypothesis is accepted). A similar comparison between the combined Al centre and single-grain TT-OSL dataset reveals dating agreement for some of the study sites, but also a tendency for the Al centre ESR ages to be significantly older than the paired TT-OSL ages, as well as associated Ti-Li centre ages or other independent age control, in some depositional contexts (Figure 5b; Table 7). These mixed comparative dating results are borne out by the regression analyses and paired statistical tests. The Al centre and single-grain TT-OSL ages display more noticeable scatter and are weakly correlated $\left(\mathrm{R}^{2}=\right.$ 0.556) when fitted with a weighted linear function (Figure 5b). According to the paired homogeneity test, only $41 \%$ of the $\mathrm{Al}$ centre ages are in agreement with their single-grain TT-OSL counterparts at $\pm 2 \sigma$. When analysed as a combined dataset, the Al centre and single-grain TT-OSL ages are considered to be statistically different from each other at the $95.4 \%$ confidence level $\left(t_{o b s}=2.88<t_{\text {crit }(\alpha: 0.05)}= \pm 2.16\right)$. 
Additional studies are clearly needed to increase the number and temporal coverage of these comparative ESR - TT-OSL datasets. However, our initial synthesis appears to highlight a potentially closer relationship between the ages of the TT-OSL and the ESR Ti-Li centre signals, rather than between the TT-OSL and Al centre signals. These datasets also reinforce the usefulness of employing the MC dating approach and emphasise the value of undertaking comparisons with semi-independent quartz dating signals, particularly single-grain TTOSL dating, in order to maximise the reliability of ESR quartz chronologies.

\subsection{Refining the chronology framework of Porto Maior using Bayesian modelling}

The Bayesian modelling results obtained in this study have enabled us to refine the scope of the existing luminescence-ESR dating framework for Porto Maior by deriving more statistically meaningful combined ages for individual depositional units at the site, and, most importantly, by permitting a revision of the existing age estimate for the in situ archaeological layer (PM4) presented by Méndez-Quintas et al. (2018). Additionally, our Bayesian modelling results at Porto Maior have provided insights into the relative merits of employing different schemes for dealing with shared dating uncertainties in OSL and ESR chronological modelling applications, which may be of practical use for similar quartz dating studies performed at other archaeological sites.

While our Bayesian modelling results show that the prior and posterior modelled age ranges of individual dating likelihood estimates are generally not significantly different from each other at the 95.4\% C.I. (Figure 5; Table 4), the modelled boundary age ranges for the various depositional units nevertheless provide a stronger statistical foundation for improved chronostratigraphic correlations of geographically disparate archaeological deposits across different parts of the Miño River basin. With considerable efforts currently ongoing to systematically date similar Acheulean deposits elsewhere in the Miño basin using ESR and luminescence techniques, (e.g. Méndez-Quintas et al., submitted), such improved chronostratigraphic correlations will be critical for future reconstructions of human occupation dynamics across the region during the Middle Pleistocene.

The results of the difference and date query analysis on the Model 1 posterior boundary probabilities reveals that the lowermost archaeological level (Unit PM3) was deposited between 252.0-276.9 ka and 239.3-263.5 ka (68.2\% boundary probability ranges) with a mean depositional age of $258.3 \pm 12.1 \mathrm{ka}$ (Figure 4; Table 5). The uppermost archaeological level, which contains the in situ LCT accumulations, was deposited between 218.9$245.9 \mathrm{ka}$ and $179.3-223 \mathrm{ka}$, with a mean depositional age of $210.7 \pm 24.7 \mathrm{ka}$. The basal sterile fluvial deposits (Unit 1-2) have lower and upper boundary age ranges of 283.8-424.0 ka and 262.9-296.5 ka, respectively, and a mean depositional age of $312.6 \pm 32.6 \mathrm{ka}$. While the uppermost unit (PM5), which is devoid of in situ artefacts, was deposited between 15.9-30.0 ka and 7.8-17.4 ka, and has a mean depositional age of $18.8 \pm 7.7$ ka. Application of the difference query to the posterior probabilities of the PM1-2 and PM3 boundaries, and the PM3 and PM4 boundaries, confirms the absence of any statistically significant temporal gap between these two groups of successive units at the $95.4 \%$ probability ranges (Table 5). This observation suggests that the preserved contacts between these units spanned relatively short timeframes that cannot be fully resolved beyond the modelled likelihood uncertainty ranges. The only statistically significant temporal hiatus identified in the 
depositional sequence took place between the accumulation of in situ archaeological unit PM4 and the overlying sterile layer (PM5). The difference query indicates a mean temporal gap of $161.7 \pm 36.4$ ka between these two units, which confirms a prolonged period of non-deposition and / or a statistically significant amount of eroded material in this part of the profile between MIS 6 to MIS 3 .

The ages for the lower sedimentary sequence at Porto Maior, with associated LFB Acheulean industry in levels PM3 and PM4, confirm the existence of this technocomplex during the Late Middle Pleistocene in the Iberian Peninsula, which is similar to that of other Iberian Acheulean sites dated to MIS 9-6 (Santonja et al., 2016), including Pinedo (López-Recio et al., 2015), Torralba (Santonja et al., 2016), Valdocarros (Rubio-Jara et al., 2016; Moreno et al., in press). The Bayesian modelled age for the in situ Porto Maior LCT accumulation (211 \pm $25 \mathrm{ka}$ at $1 \sigma$ ) overlaps with, but is slightly younger than, the age range of 293-205 ka originally inferred by Méndez Quintas et al. (2018), which was based on the $2 \sigma$ confidence interval of the weighted mean Ti centre and pIR-IRSL ages for units PM3-PM4. The Bayesian modelling chronostratigraphic framework therefore suggests the in situ lithic-bearing deposits at the base of Unit PM4 most likely accumulated during MIS 7 rather than during MIS 7-8 as originally proposed by Méndez-Quintas et al. (2018). These results also confirm the presence of LFB Acheulean at a time when non-LFB Acheulean and early Middle Palaeolithic assemblages are found elsewhere on the Iberian Peninsula (Santonja et al., 2014).

\section{Conclusion}

This study has added several new facets to our chronological understanding of the Porto Maior archaeological site: (i) It has enhanced the scope of the original dating framework presented by Méndez-Quintas et al. (2018) via the establishment of new single-grain TT-OSL ages for level PM3 and (ii) it has derived more statistically meaningful combined age ranges for the various depositional units and archaeological finds at the site using an integrated Bayesian modelling approach. In doing so it has confirmed the suitability of the original ESR MC dating interpretations of Méndez-Quintas et al. (2018), and enabled refinement of the depositional age for the in situ LCT accumulation at Porto Maior $(210.7 \pm 24.7 \mathrm{ka}$ at $1 \sigma)$.

The results of this study suggest a potentially close relationship between TT-OSL and Ti-Li paramagnetic centres. This interpretation appears to be supported by our synthesis of comparative ESR-single-grain TT-OSL dating studies performed at other sites. From an ESR dating perspective, our new results and analyses of published datasets imply that the MC dating approach is likely to be advantageous in many dating contexts and that there is good potential for obtaining reliable Middle to Early Pleistocene chronologies using appropriate ESR quartz dating methodologies; especially where they are applied in combination with other extended-range quartz dating techniques such as single-grain TT-OSL.

\section{Acknowledgments}

M.D. is funded by Australian Research Council (ARC) Discovery Early Career Researcher Award DE160100743 and L.J.A. is funded by ARC Future Fellowship project FT130100195. M. Duval is funded by ARC Future Fellowship Grant FT150100215. 


\section{References}

Adamiec, G., Duller, G.A.T., Roberts, H.M., Wintle, A.G., 2010. Improving the TT-OSL SAR protocol through source trap characterisation. Radiation Measurements 54, 768-777.

Arnold, L.J., Roberts, R.G., 2009. Stochastic modelling of multi-grain equivalent dose $\left(\mathrm{D}_{\mathrm{e}}\right)$ distributions: implications for OSL dating of sedimentmixtures. Quaternary Geochronology 4, 204-230.

Arnold, L.J., Roberts, R.G., 2011. Paper I - Optically stimulated luminescence (OSL) dating of perennially frozen deposits in north-central Siberia: OSL characteristics of quartz grains and methodological considerations regarding their suitability for dating. Boreas 40, 389-416.

Arnold, L.J., Demuro, M., Navazo Ruiz, M., Benito-Calvo, A., Pérez-González, A., 2013. OSL dating of the Middle Palaeolithic Hotel California site, Sierra de Atapuerca, northcentral Spain. Boreas 42, 285-305.

Arnold, L.J., Demuro, M., Parés, J.M., Arsuaga, J.L., Aranburu, A., Bermúdez de Castro, J.M., Carbonell, E., 2014. Luminescence dating and palaeomagnetic age constraint on hominins from Sima de los Huesos, Atapuerca, Spain. Journal of Human Evolution 67, 85-107.

Arnold, L.J., Demuro, M., Parés, J.M., Pérez-González, A., Arsuaga, J.L., Bermúdez de Castro, J.M., Carbonell, E., 2015. Evaluating the suitability of extended-range luminescence dating techniques over Early and Middle Pleistocene timescales: published datasets and case studies from Atapuerca, Spain. Quaternary International 389, 167-190.

Arnold, L.J., Demuro, M., Spooner, N.A., Prideaux, G.J., McDowell, M.C., Camens, A.B., Reed, E.H., Parés, J.M., Arsuaga, J.L., Bermúdez de Castro, J.M., Carbonell, E., 2019. Single-grain TT-OSL bleaching characteristics: insights from modern analogues and OSL dating comparisons. Quaternary Geochronology 49, $45-51$.

Arnold, L.J., Demuro, M., 2015. Insights into TT-OSL signal stability from single-grain analyses of known-age deposits at Atapuerca, Spain. Quaternary Geochronology 30B, 472-478.

Arriolabengoa, M., Iriarte, E., Aranburu, A., Yusta, I., Arnold, L.J., Demuro, M., Arrizabalaga, A., 2018. Reconstructing the sedimentary history of Lezetxiki II cave (Basque Country, northern Iberian Peninsula) using micromorphological analysis. Sedimentary Geology 372, 96-111.

Arsuaga, J.L., Martínez, I., Arnold, L.J., Aranburu, A., Gracia-Téllez, A., Sharp, W.D., Quam, R.M., Falguères, C., Pantoja-Pérez, A., Bischoff, J., Poza-Rey, E., Parés, J.M., Carretero, J.M., Demuro, M., Lorenzo, C., Sala, N., Martinón-Torres, M., García, N., Alcázar de Velasco, A., Cuenca-Bescós, G., Gómez-Olivencia, A., 
Moreno, D., Pablos, A., Shen, C.-C., Rodríguez, L., Ortega, A.I., García, R., Bonmatí, A., Bermúdez de Castro, J.M., Carbonell, E., 2014. Neandertal roots: cranial and chronological evidence from Sima de los Huesos. Science 344, 1358-1363.

Bahain et al., this volume

Bartz, M., Rixhon, G., Duval, M., King, G.E., Álvarez Posada, C., Parés, J.M., Brückner, H., 2018. Successful combination of electron spin resonance, luminescence and palaeomagnetic dating methods allows reconstruction of the Pleistocene evolution of the lower Moulouya river (NE Morocco). Quaternary Science Reviews 185, 153-171.

Bartz, M., Arnold, L.J., Demuro, M., Duval, M., King, G.E., Rixhon, G., Álvarez Posada, C., Parés, J.M., Brückner, H., 2019. Single-grain TT-OSL dating results confirm an Early Pleistocene age for the lower Moulouya River deposits (NE Morocco). Quaternary Geochronology 49, 138-145.

Beerten, K., Lomax, J., Clémer, K., Stesmans, A., Radtke, U., 2006. On the use of Ti centres for estimating burial ages of Pleistocene sedimentary quartz: Multiple-grain data from Australia. Quaternary Geochronology $1,151-158$.

Brennan, B. J. Beta doses to spherical grains. Radiation Measurements 37, 299-303,

Bronk Ramsey, C., 2009a. Bayesian analysis of radiocarbon dates. Radiocarbon 51, 337-360.

Bronk Ramsey, C., 2009b. Dealing with offsets and outliers in radiocarbon dating. Radiocarbon 51, $1023-1045$.

Bronk Ramsey, C., Albert, P.G., Blockley, S.P.E., Hardiman, M., Housley, R.A., Lane, C.S., Lee, S., Matthews, I.P., Smith, V.C., Lowe, J.J., 2015. Improved age estimates for key Late Quaternary European tephra horizons in the RESET lattice. Quaternary Science Reviews 118, 18-32.

del Val, M., Duval, M., Medialdea, A., Bateman, M.D., Moreno, D., Arriolabengoa, M., Aranburu, A., Iriarte, E., 2019. First chronostratigraphic framework of fluvial terrace systems in the eastern Cantabrian margin (Bay of Biscay, Spain). Quaternary Geochronology 49, 108-114.

Demuro, M., Arnold, L.J., Froese, D.G., Roberts, R.G., 2013. OSL dating of loess deposits bracketing Sheep Creek tephra beds, northwest Canada: Dim and problematic single-grain OSL characteristics and their effect on multi-grain age estimates. Quaternary Geochronology 15, 67-87.

Demuro, M., Arnold, L.J., Parés, J.M., Pérez-González, A., Ortega, A.I., Arsuaga, J.L., Bermúdez de Castro, J.M., Carbonell, E., 2014. New luminescence ages for the Galería Complex archaeological site: Resolving 
chronological uncertainties on the Acheulean record of the Sierra de Atapuerca, northern Spain. PLoS One 9, e110169.

Demuro, M., Arnold, L.J., Parés, J.M., Sala, R., 2015. Extended-range luminescence chronologies suggest potentially complex bone accumulation histories at the Early-to-Middle Pleistocene palaeontological site of Huéscar-1 (Guadix-Baza basin, Spain). Quaternary International 389, 191-212.

Demuro, M., Arnold, L.J., Aranburu, A., Sala, N., Arsuaga, J.L., 2019. New bracketing luminescence ages constrain the Sima de los Huesos hominin fossils (Atapuerca, Spain) to MIS 12. Journal of Human Evolution, accepted.

Duller, G.A.T., 2003. Distinguishing quartz and feldspar in single grain luminescence measurements. Radiation Measurements 37, 161-165.

Duller, G.A.T., Wintle, A.G., 2012. A review of the thermally transferred optically stimulated luminescence signal from quartz for dating sediments. Quaternary Geochronology 7, 6-20.

Duval, M. Guilarte, V., 2015. ESR dosimetry of optically bleached quartz grains extracted from PlioQuaternary sediment: Evaluating some key aspects of the ESR signals associated to the Ti-centers. Radiation Measurements 78, 28-41.

Duval, M., Sancho, C., Calle, M., Guilarte, V., Peña-Monné, J.L., 2015. On the interest of using the multi center approach in ESR dating of optically bleached quartz grains: some examples from the Early Pleistocene terraces of the Alcanadre River (Ebro basin, Spain). Quaternary Geochronology 29, 58-69.

Duval, M., Arnold, L. J., Guilarte, V., Demuro, M., Santonja, M., Pérez-González, A. 2017. Electron spin resonance dating of optically bleached quartz grains from the Middle Palaeolithic site of Cuesta de la Bajada (Spain) using the multiple centres approach. Quaternary Geochronology 37, 82-96.

Duval, M., Grün, R., Parés, J.M., Martín-Francés, L., Campaña, I., Rosell, J., Shao, Q., Arsuaga, J.L., Carbonell, E. and Bermúdez de Castro, J.M. 2018. The first direct ESR analysis of a hominin tooth from Atapuerca Gran Dolina TD-6 (Spain) supports the antiquity of Homo antecessor. Quaternary Geochronology 47, pp. 120-137. 10.1016/j.quageo.2018.05.001.

Duval, M., Voinchet, P., Arnold, L.J., Parés, J.M., Minnella, W., Guilarte, V., Demuro, M., Falguères, C., Bahain, J.-J., Despriée, J., this volume. A multi-technique dating study of two Lower Palaeolithic sites from the Middle Loire Basin, France: Lunery-la Terre-des-Sablons and Brinay-la Noira. Quaternary International. 
Falguères, C., 1986. Datation de sites acheuléens et moustériens du Midi méditerranéen par la méthode de résonance de spin électronique. Ph.D. Thesis, Muséum National d'Histoire Naturelle. 173 p.

Falguères, C., Yokoyama, Y. ,Miallier, D., 1991. Stability of some centres in quartz. International Journal of Radiation Applications and Instrumentation. Part D. Nuclear Tracks and Radiation Measurements 18, $155-161$.

Falguères, C., Bahain, J.-J., Bischoff, J.L., Pérez-González, A., Ortega, A.I., Ollé, A., Quiles, A., Ghaleb, B., Moreno, D., Dolo, J.-M., Shao, Q., Vallverdú, J., Carbonell, E., Bermúdez de Castro, J.M., Arsuaga, J.L., 2013. Combined ESR/U-series chronology of Acheulian hominid-bearing layers at Trinchera Galería site, Atapuerca, Spain. Journal of Human Evolution 65, 168-184.

Galbraith, R.F., Roberts, R.G., Laslett, G.M., Yoshida, H., Olley, J.M., 1999. Optical dating of single and multiple grains of quartz from Jinmium rock shelter, northern Australia: part I, experimental design and statistical models. Archaeometry 41, 339-364.

Galbraith, R., 2003. A simple homogeneity test for estimates of dose obtained using OSL. Ancient TL 21, 7577.

Galbraith, R.F., Roberts, R.G., 2012. Statistical aspects of equivalent dose and error calculation and display in OSL dating: An overview and some recommendations. Quaternary Geochronology 11, 1-17.

Gao, L., Yin, G.-M., Liu, C.-R., Bahain, J.-J., Lin, M., Li, J.-P., 2009. Natural sunlight bleaching of the ESR titanium centre in quartz. Radiation Measurements 501-504.

Grün, R., 1989. Electron spin resonance (ESR) dating. Quaternary International 1, 65-109.

Guérin, G., Mercier, M., Adamiec, G., 2011. Dose-rate conversion factors: update. Ancient TL 29, 5-8.

Hamm, G., Mitchell, P., Arnold, L.J., Prideaux, G.J., Questiaux, D., Spooner, N.A., Levchenko, V.A., Foley, E.C., Worthy, T.H., Stephenson, B., Coulthard, V., Coulthard, C., Wilton, S., Johnston, D., 2016. Cultural innovation and megafauna interaction in the early settlement of arid Australia. Nature 539, 280-283.

Higham, T., Douka, K., Wood, R., Bronk Ramsey, C., Brock, F., Basell, L., Camps, M., Arrizabalaga, A., Baena, J., Barroso-Ruíz, C., Bergman, C., Boitard, C., Boscato, P., Caparrós, M., Conard, N.J., Draily, C., Froment, A., Galván, B., Gambassini, P., Garcia-Moreno, A., Grimaldi, S., Haesaerts, P., Holt, B., IriarteChiapusso, M.-J., Jelinek, A., Jordá Pardo, J.F., Maíllo-Fernández, J.-M., Marom1, A., Maroto, J., Menéndez, M., Metz, L., Morin, E., Moroni, A., Negrino, F., Panagopoulou, E., Peresani, M., Pirson, S., de la Rasilla, M., Riel-Salvatore, J., Ronchitelli, A., Santamaria, D., Semal, P., Slimak, L., Soler, J., Soler, N., Villaluenga, A., 
Pinhasi, R., Jacobi, R., 2014. The timing and spatiotemporal patterning of Neanderthal disappearance. Nature 512, 306-309.

Hogg, A.G., Hua, Q., Blackwell, P.G., Niu, M., Buck, C.E., Guilderson, T.P., Heaton, T.J., Palmer, J.G., Reimer, P.R., Reimer, R.W., Turney, C.S.M., Zimmerman, S.R.H., 2013. SHCal13 Southern Hemisphere calibration 0-50,000 years cal BP. Radiocarbon 55, 1889-1903.

Ingicco, T., van den Bergh, G.D., Jago-on, C., Bahain, J.J., Chacón, M.G., Amano, N., Forestier, H., King, C., Manalo, K., Nomade, S., Pereira, A., Reyes, M.C., Sémah, A.M., Shao, Q., Voinchet, P., Falguères, C., Albers, P.C.H., Lising, M., Lyras, G., Yurnaldi, D., Rochette, P., Bautista, A. and de Vos, J., 2018. Earliest known hominin activity in the Philippines by 709 thousand years ago. Nature 557, 233-237.

Jacobs, Z., Roberts, R.G., Lachlan, T.J., Karkanas, P., Marean, C.W., Roberts, D.L., 2011. Development of the SAR TT-OSL procedure for dating Middle Pleistocene dune and shallow marine deposits along the Cape coast of South Africa. Quaternary Geochronology 6, 491-513.

Kennett, J.P., Kennett, D.J., Culleton, B.J., Emili Aura Tortosa, J., Bischoff, J.L., Bunch, T.E., Randolph Daniel Jr., I., Erlandson, J.M., Ferraro, D., Firestone, R.B., Goodyear, A.C., Israde-Alcántara, I., Johnson, Jordá Pardo, J.F., Kimbel, D.R., LeCompte, M.A., Lopinot, N.H., Mahaney, W.C., Moore, A.M.T., Moore, C.R., Ray, J.H., Stafford Jr., T.W., Barnett Tankersley, K., Wittke, J.H., Wolbach, W.S., West, A., 2015. Bayesian chronological analyses consistent with synchronous age of 12,835-12,735 Cal B.P. for Younger Dryas boundary on four continents. Proceedings of the National Academy of Sciences of the USA 112, E4344-E4353.

Laurent, M., 1993. Datation par résonance de spin électronique (ESR) de quartz de formations quaternaires : comparaison avec le paléomagnétisme. Ph.D. Thesis, Muséum National d'Histoire Naturelle. 104 p.

Liu, C.R., Yin, G.M., Fang, F., Voinchet, P., Deng, C.L., Han, F., Li, J.P., Song, W.J., Wang, D., Bahain, J.-J., 2013. ESR dating of the Donggutuo Palaeolithic site in the Nihewan Basin, northern China. Geochronometria 40, 348-354.

Liu, C.-R., Yin, G.-M., Demg, C.-L., Han, F., Song, W.-J., 2014. ESR dating of the Majuangou and Banshan Paleolithic sites in the Nihewan Basin, North China. Journal of Human Evolution 73, 58-63.

Mayya, Y.S., Morthekai, P., Murari, M.K., Singhvi, A.K., 2006. Towards quantifying beta microdosimetric effects in single-grain quartz dose distribution. Radiation Measurements 41, 1032-1039.

Macken, A.C., Staff, R.A., Reed, E.H., 2013. Bayesian age-depth modelling of Late Quaternary deposits from Wet and Blanche Caves, Naracoorte, South Australia: A framework for comparative faunal analyses. Quaternary Geochronology 17, 26-43. 
Méndez-Quintas, E., Santonja, M., Pérez-González, A., Duval, M., Demuro, M., Arnold, L.J., 2018. First evidence of an extensive Acheulean large cutting tool accumulation in Europe from Porto Maior (Galicia, Spain). Scientific Reports 8, 3082.

Méndez-Quintas, E., Demuro, M., Arnold, L.J., Duval, M., Pérez-González, A., Santonja, M., in press. Insights into the late stages of the Acheulean technocomplex of Western Iberia from the Arbo site (Galicia, Spain). Journal of Archaeological Science: Reports.

Moreno, D., Falguères, C., Pérez-González, A., Duval, M., Voinchet, P., Benito-Calvo, A., Ortega, A.I., Bahain, J.-J., Sala, R., Carnobell, E., Bermúdez de Castro, J.M., Arsuaga, J.-L., 2012. ESR chronology of alluvial deposits in the Arlanzón valley (Atapuerca, Spain): Contemporaneity with Atapuerca Gran Dolina site. Quaternary Geochronology 10, 418-423.

Moreno, D., Falguères, C., Pérez-González, A., Voinchet, P., Ghaleb, B., Despriée, J., Bahain, J.-J., Sala, R., Carbonell, E., Bermúdez de Castro, M.J., Arsuaga, J.L., 2015. New radiometric dates on the loest stratigraphical section (TD1 to TD6) of Gran Dolina site (Atapuerca, Spain). Quaternary Geochronology 30, 535-540.

Nathan, R.P., Thomas, P.J., Jain, M., Murray, A.S., Rhodes, E.J., 2003. Environmental dose rate heterogeneity of beta radiation and its implications for luminescence dating: Monte Carlo modelling and experimental validation. Radiation Measurements 37, 305-313.

Parés, J.M., Arnold, L.J., Duval, M., Demuro, M., Pérez-González, A., Bermúdez de Castro, J.M., Carbonell, E., Arsuaga, J.L., 2013. Reassessing the age of Atapuerca-TD6 (Spain): new paleomagnetic results. Journal of Archaeological Science 40, 4586-45495.

Pereira, A., S. Nomade, M.-H. Moncel, P. Voinchet, J.-J. Bahain, I. Biddittu, C. Falguères, B. Giaccio, G. Manzi, F. Parenti, G. Scardia, V. Scao, G. Sottili and A. Vietti, 2018. Integrated geochronology of Acheulian sites from the southern Latium (central Italy): Insights on human-environment interaction and the technological innovations during the MIS 11-MIS 10 period. Quaternary Science Reviews 187, 112-129.

Porat, N., Duller, G.A.T., Roberts, H.M., Wintle, A.G., 2009. A simplified SAR protocol for TT-OSL. Radiation Measurements 44, 538-542.

Preusser, F., Chithambo, M.L., Götte, T., Martini, M., Ramseyer, K., Sendezera, E.J., Susino, G.J., Wintle, A.G., 2009. Quartz as a natural luminescence dosimeter. Earth-Science Reviews 97, 184-214. 
Prescott, J.R., Hutton, J.T., 1994. Cosmic ray contributions to dose rates for luminescence and ESR dating: large depths and long-term time variations. Radiation Measurements 23, 497-500.

Reimer, P.J., Reimer, R.W., 2001. A marine reservoir correction database and on-line interface. Radiocarbon $43,461-463$.

Rhodes, E.J., Bronk Ramsey, C., Outram, Z., Batt, C., Willis, L., Dockrill, S. and Bond, J., 2003. Bayesian methods applied to the interpretation of multiple OSL dates: high precision sediment ages from Old Scatness Broch excavations, Shetland Isles. Quaternary Science Reviews 22, 1231-1244.

Rink, W.J., Bartoll, J., Schwarcz, H.P., Shane, P., Bar-Yosef, O., 2007. Testing the reliability of ESR dating of optically exposed buried quartz sediments. Radiation Measurements 42, 1618-1626.

Sahnouni, M., Pares, J.M., Duval, M., Caceres, I., Harichane, Z., van der Made, J., Perez-Gonzalez, A., Abdessadok, S., Kandil, N., Derradji, A., Medig, M., Boulagraif, K., Semaw, S., 2018. 1.9-million- and 2.4million-year-old artifacts and stone tool-cutmarked bones from Ain Boucherit, Algeria. Science. 10.1126/science.aau0008 (2018).

Santonja, M., Pérez-González, A., Domínguez-Rodrigo, M., Panera, J., Rubio-Jara, S., Sesé, C., Soto, E., Arnold, L.J., Duval, M., Demuro, M., Ortiz, J.E., de Torres, T., Mercier, N., Barba, R., Yravedra, J., 2014. The Middle Paleolithic site of Cuesta de la Bajada (Terual, Spain): a perspective on the Acheulean and Middle Paleolithic technocomplexes in Europe. Journal of Archaeological Science 49, 556-571.

Santonja, M., Pérez-González, A., Panera, J., Rubio-Jara, S., Méndez-Quintas, E., 2016. The coexistence of Acheulean and Ancient Middle Palaeolithic techno-complexes in the Middle Pleistocene of the Iberian Peninsula. Quaternary International 411, 367-377.

Scott, E.M., Cook, G.T., Naysmith, P., 2007. Error and uncertainty in radiocarbon measurements. Radiocarbon $49,427-440$.

Stevens, T., Buylaert, J.-P., Murray, A.S., 2009. Towards development of a broadly-applicable SAR TT-OSL dating protocol for quartz. Radiation Measurements 44, 639-645.

Stuiver M, Polach H., 1977. Discussion: reporting of ${ }^{14} \mathrm{C}$ data. Radiocarbon 19, 355-363.

Tanaka, K., Machette, M.N., Crone, A.J., Roger Bowman, J., 1995. ESR dating of aeolian sand near Tennant creek, Northern territory, Australia. Quaternary Science Reviews 14, 385-393.

Tissoux, H., Falguères, C., Voinchet, P., Toyoda, S., Bahain, J.J., Despriée, J., 2007. Potential use of Ti-center in ESR dating of fluvial sediment. Quaternary Geochronology 2, 367-372. 
Tissoux H., Toyoda S., Falguères F., Voinchet P., Takada M., Bahain J.-J., Despriée, J., 2008. ESR dating of sedimentary quartz from two Pleistocene deposits using Al and Ti-centers. Geochronometria 30, 23-31.

Toyoda, S., Ikeya, M., 1991. Thermal stabilities of paramagnetic defect and impurity centers in quartz: Basis for ESR dating of thermal history. Geochemical Journal 25, 437-445.

Toyoda, S., Voinchet, P., Falguères, C., Dolo, J. M., Laurent, M., 2000. Bleaching of ESR signals by the sunlight: a laboratory experiment for establishing the ESR dating of sediments. Applied Radiation and Isotopes $52,1357-1362$.

Tsukamoto, S., Duller, G.A.T., Wintle, A.G., 2008. Characteristics of thermally transferred optically stimulated luminescence (TT-OSL) in quartz and its potential for dating sediments. Radiation Measurements 43, 12041218.

Voinchet, P., 2002. Datation par résonance paramagnétique électronique (RPE) de quartz blanchis extraits de sédiments fluviatiles pléistocènes: contribution méthodologique et application aux systèmes de la Creuse, du Loir et de l'Yonne. Ph.D. Thesis, Muséum National d'Histoire Naturelle, Paris. 234 + XCIV p.

Voinchet, P., Falguères, C., Laurent, M., Toyoda, S., Bahain, J.J., Dolo, J.M., 2003. Artificial optical bleaching of the Aluminium center in quartz implications to ESR dating of sediments. Quaternary Science Reviews 22, $1335-1338$.

Voinchet, P., Bahain, J.-J., Falguères, C., Laurent, M., Dolo, J.-M., Despriée, J., Gageonnet, R., Chaussé, C., 2004. ESR dating of quartz extracted from Quaternary sediments application to fluvial terraces system of northern France. Quaternaire 15, 135-141.

Voinchet, P., Moreno, D., Bahain, J.-J., Tissoux, H., Tombret, O., Falguères, C., Moncel, M.-H., Schreve, D., Candy, I., Antoine, P., Ashton, N., Beamish, M., Cliquet, D., Despriée, J., Lewis, S., Limondin-Lozouet, L., Locht, J.-L., Parfitt, S., Pope, M., 2015. New chronological data (ESR and ESR/U-series) for the earliest Acheulian sites of north-western Europe. Journal of Quaternary Science 30, 610-622.

Voinchet, P., Pereira, A., Nomade, S., Falguères, C., Biddittu, I., Piperno, M., Moncel, M.-H., Bahain, J.-J. ESR dating applied to optically bleached quartz - a comparison with ${ }^{40} \mathrm{Ar} /{ }^{39} \mathrm{Ar}$ chronologies on Italian Middle Pleistocene sequences. Quaternary International, this volume.

Walther, R., Zilles, D., 1994. ESR studies on bleached sedimentary quartz. Quaternary Science Reviews 13, $611-614$. 
Wang, X.L., Lu, Y.C., Wintle, A.G., 2006. Recuperated OSL dating of fine-grained quartz in Chinese loess. Quaternary Geochronology 1, 89-100.

Wang, X.L., Wintle, A.G., Lu, Y.C., 2007. Testing a single-aliquot protocol for recuperated OSL dating. Radiation Measurements 42, 380-391.

Wintle, A.G., Adamiec, G., 2017. Optically stimulated luminescence signals from quartz: A review. Radiation Measurements 98, 10-33.

Yokoyama, Y., Falguères, C., Quaegebeur, J.P., 1985. ESR dating of quartz from quaternary sediments: first attempt. Nuclear Tracks and Radiation Measurements 10, 921-928.

Yoshida, H., 1996. Quaternary dating studies using ESR signals, with emphasis on shell, coral, tooth enamel and quartz. Ph.D. Thesis, Australian National University, Canberra.

\section{Figure Captions}

Figure 1. (A) Location of Porto Maior (black star), Galicia, Spain. (B) Stratigraphic section showing the previously published pIR-IRSL, single-grain OSL and ESR ages for Porto Maior, together with the new TTOSL ages obtained in the present study (modified from Méndez-Quintas et al., 2018).

Figure 2. (A, B) Example of a sensitivity-corrected TT-OSL dose-response and decay curve for an individual grain from sample PM16-3. White square denotes the sensitivity-corrected natural OSL signal; filled circles denote the sensitivity-corrected regenerated OSL signals; white circle denotes the repeated regenerative dose point used to calculate the recycling ratio. The $\mathrm{D}_{0}$ value characterises the rate of signal saturation with respect to administered dose and equates to the dose value for which the saturating exponential dose-response curve slope is $1 / \mathrm{e}$ (or 0.37) of its initial value. $(\mathrm{C}-\mathrm{F})$ The single-grain TT-OSL $\mathrm{D}_{\mathrm{e}}$ distributions obtained for samples PM16-4, PM16-6, PM16-5 and PM16-3. The grey bars on the radial plots are centred on the central age model (CAM) $D_{e}$ value used to derive the final burial dose estimate for each sample. Also shown are the overdispersion (OD) values associated with each $D_{e}$ dataset.

Figure 3. Bayesian modelling results for the Porto Maior stratigraphic sequence (Model 1; see main text). The prior age distributions for the dating samples (likelihoods) are shown as light blue probability density functions (PDFs). The modelled posterior distributions for the dating samples and stratigraphic unit boundaries are shown as dark blue and grey PDFs, respectively. Unmodelled and modelled ages are shown on a calendar year timescale and both are expressed in years before AD2014 (average sample collection date). The white circles and associated error bars represent the mean ages and $1 \sigma$ uncertainty ranges of the PDFs. The $68.2 \%$ and $95.4 \%$ ranges of the highest posterior probabilities are indicated by the horizontal bars underneath the PDFs. 
Figure 4. Bayesian-modelled durations of the stratigraphic units at Porto Maior (Model 1; see main text). The PDFs have been calculated from the modelled posterior probabilities of the upper and lower boundaries of each stratigraphic unit (shown as grey PDFs in Figure 5) using the date query function in OxCal v4.3.2.

Figure 5. ESR and TT-OSL age comparisons based on published dating studies conducted on a range of sites from Spain, France and Morocco (based on data shown in Table 7). The uncertainties for each age estimate represent the $1 \sigma$ errors. The type of ESR centre used to derive comparative ages for each TT-OSL sample correspond to the Ti-Li centre (option D) in plot (A) and the Al centre in plot (B). Each dataset has been fitted with a linear least-squares function (dashed line) weighted according to the inverse squared relative errors of both coordinates, following the approach of Reed (1989). The confidence intervals about the linear regression functions (long-dashed lines) have been calculated using the Student's t-statistic for a two-tailed test with (n-2) degrees of freedom and are presented at the $95.4 \%$ confidence level $(\alpha=0.05)$. The solid line represents the 1:1 line for each dataset. 


\begin{tabular}{|c|c|c|c|c|c|c|c|c|}
\hline \multirow[b]{2}{*}{ Level } & \multirow[b]{2}{*}{ Sample } & \multicolumn{3}{|l|}{ ESR age $(\mathrm{ka})$} & \multicolumn{4}{|c|}{ Luminescence age (ka) } \\
\hline & & $\mathrm{Al}$ centre & Ti-Li centre (op. D) & Ti-H centre & Sample & Single-grain OSL & pIR-IRSL 225 & Single-grain TT-OSL \\
\hline PM5 & MIN1401 & $138.5 \pm 25.2(23.3)$ & $124.2 \pm 12.2(8.5)$ & $47.5 \pm 10.0(9.5)$ & PM16-4 & $17.1 \pm 1.3(1.0)$ & $16.6 \pm 0.9(0.5)$ & $25.8 \pm 3.4(3.2)$ (This study) \\
\hline PM4 & MIN1402 & $181.5 \pm 24.0(19.6)$ & $205.5 \pm 20.0(12.3)$ & & PM16-6 & & $230.6 \pm 14.8(7.7)$ & $172.1 \pm 20.5(17.6)($ This study) \\
\hline PM4 & VI1206 & $349.7 \pm 45.7(37.2)$ & $237.8 \pm 21.1(10.5)$ & & & & & \\
\hline PM3 & VI1204 & $441.3 \pm 74.8(66.8)$ & $278.8 \pm 26.1(15.0)$ & & PM16-5 & & $268.3 \pm 24.0(19.8)$ & $225.3 \pm 30.6(27.9)$ (This study) \\
\hline PM3 & VI1205 & $452.4 \pm 66.4(56.8)$ & $265.5 \pm 22.9(10.4)$ & & PM16-3 & & $259.1 \pm 28.8(24.4)$ & $241.5 \pm 32.2(28.0)$ (This study) \\
\hline PM1 & MIN1403 & $424.4 \pm 85.1(78.7)$ & $264.3 \pm 26.8(18.0)$ & & & & & \\
\hline
\end{tabular}

Table 1. ESR (quartz) and luminescence chronologies (quartz and potassium feldspars) obtained previously for Porto Maior, Spain (Méndez-Quintas et al., 2018). The new single-grain TT-OSL quartz ages obtained in this study are also provided in the last column. The dating uncertainties shown outside parentheses represent the total (random plus systematic) $1 \sigma$ range for each sample, whereas those shown in parentheses represent the random $1 \sigma$ dating uncertainties only. 


\begin{tabular}{|c|c|c|}
\hline Step & Single-grain TT-OSL SAR protocol & Signal \\
\hline $1^{\mathrm{a}}$ & Give dose & \\
\hline 2 & Preheat to $260^{\circ} \mathrm{C}$ for $10 \mathrm{~s}$ & \\
\hline 3 & Stimulate with green laser at $125^{\circ} \mathrm{C}$ for $3 \mathrm{~s}$ ( $90 \%$ power) & \\
\hline 4 & Preheat to $260^{\circ} \mathrm{C}$ for $10 \mathrm{~s}$ & \\
\hline 5 & Stimulate with green laser at $125^{\circ} \mathrm{C}$ for $3 \mathrm{~s}$ ( $90 \%$ power) & $\begin{array}{l}\mathrm{TT}-\mathrm{OSL} \\
\mathrm{L}_{\mathrm{n}} \text { or } \mathrm{L}_{\mathrm{x}}\end{array}$ \\
\hline 6 & Stimulate with blue LEDs at $280^{\circ} \mathrm{C}$ for $400 \mathrm{~s}$ & \\
\hline 7 & Give test dose (250 Gy) & \\
\hline 8 & Preheat to $260^{\circ} \mathrm{C}$ for $10 \mathrm{~s}$ & \\
\hline 9 & Stimulate with green laser at $125^{\circ} \mathrm{C}$ for $3 \mathrm{~s}$ ( $90 \%$ power) & \\
\hline 10 & Preheat to $260^{\circ} \mathrm{C}$ for $10 \mathrm{~s}$ & \\
\hline 11 & Stimulate with green laser at $125^{\circ} \mathrm{C}$ for $3 \mathrm{~s}$ ( $90 \%$ power) & $\begin{array}{l}\text { TT-OSL } \\
\mathrm{T}_{\mathrm{n}} \text { or } \mathrm{T}_{\mathrm{x}}\end{array}$ \\
\hline 12 & Stimulate with blue LEDs at $290^{\circ} \mathrm{C}$ for $400 \mathrm{~s}$ & \\
\hline 13 & Return to 1 & \\
\hline
\end{tabular}

${ }^{\text {a }}$ Step omitted when measuring the natural signal $\left(L_{n}\right)$.

Table 2. SAR protocol used in this study to obtain single-grain quartz TT-OSL ages for the Porto Maior samples. $\mathrm{L}_{\mathrm{x}}=$ regenerative dose signal response; $\mathrm{L}_{\mathrm{n}}=$ natural dose signal response; $\mathrm{T}_{\mathrm{x}}=$ test dose signal response for a laboratory dose cycle $T_{n}=$ test dose signal response for the natural dose cycle. 


\begin{tabular}{|c|c|c|c|c|c|c|c|c|c|}
\hline Sample & $\begin{array}{l}\text { Accepted/ } \\
\text { measured }^{\text {a }}\end{array}$ & $\begin{array}{l}\text { Overdis- } \\
\text { persion } \\
(\%)^{b}\end{array}$ & $\begin{array}{l}\text { CAM De } \\
(\mathrm{Gy})\end{array}$ & $\begin{array}{l}\text { Water } \\
\text { content } \\
(\%)^{c}\end{array}$ & $\begin{array}{l}\text { Beta dose } \\
\text { rate } \\
(\mathrm{Gy} / \mathrm{ka})^{\mathrm{d}} \\
\end{array}$ & $\begin{array}{l}\text { Gamma dose } \\
\text { rate } \\
(\mathrm{Gy} / \mathrm{ka})^{\mathrm{e}}\end{array}$ & $\begin{array}{l}\text { Cosmic dose } \\
\text { rate } \\
(\mathrm{Gy} / \mathrm{ka})^{\mathrm{f}}\end{array}$ & $\begin{array}{l}\text { Total dose } \\
\text { rate } \\
(\mathrm{Gy} / \mathrm{ka})^{\mathrm{g}} \\
\end{array}$ & $\begin{array}{l}\text { Age } \\
(\mathrm{ka})^{\mathrm{h}}\end{array}$ \\
\hline PM16-4 & $27 / 2600$ & $47 \pm 12$ & $95 \pm 12$ & 15.0 & $1.99 \pm 0.05$ & $1.49 \pm 0.05$ & $0.17 \pm 0.02$ & $3.68 \pm 0.15$ & $26 \pm 3$ \\
\hline PM16-6 & $22 / 1300$ & $32 \pm 10$ & $556 \pm 56$ & 24.9 & $1.75 \pm 0.09$ & $1.30 \pm 0.05$ & $0.15 \pm 0.02$ & $3.23 \pm 0.20$ & $172 \pm 21$ \\
\hline PM16-5 & 14 / 1000 & $32 \pm 12$ & $732 \pm 89$ & 19.8 & $1.70 \pm 0.08$ & $1.38 \pm 0.05$ & $0.14 \pm 0.02$ & $3.25 \pm 0.18$ & $225 \pm 31$ \\
\hline PM16-3 & $23 / 1300$ & $38 \pm 10$ & $804 \pm 92$ & 28.5 & $1.80 \pm 0.09$ & $1.39 \pm 0.05$ & $0.11 \pm 0.01$ & $3.33 \pm 0.22$ & $242 \pm 32$ \\
\hline
\end{tabular}

a Number of $D_{e}$ measurements that passed the standard SAR rejection criteria / total number of $D_{e}$ measured made per sample.

$\mathrm{b}$ The relative spread in the $\mathrm{D}_{\mathrm{e}}$ dataset beyond that associated with the measurement uncertainties for individual $\mathrm{D}_{\mathrm{e}}$ values, calculated using the central age model $(\mathrm{CAM})$ of Galbraith et al. (1999).

${ }^{c}$ Water content used for calculating environmental dose rates, expressed as $\%$ of dry mass of sample and assigned a relative uncertainty of $\pm 20 \%$. For samples PM16-3, PM16-5 and PM16-6 the long-term water contents are taken as being equivalent to 50\% of saturated values, while for sample PM16-4 the water content was set to 15\%

(see justification in Méndez-Quintas et al., 2018). An associated uncertainty of $20 \%$ was added to water content values when calculating dose rates.

${ }^{\mathrm{d}}$ Beta dose rates were calculated using a Ris $\varnothing$ GM-25-5 low-level beta counter (Bøtter-Jensen and Mejdahl, 1988), after making allowance for beta dose attenuation due to grain-size effects and HF etching (Brennan, 2003).

e Gamma dose rates were calculated from in situ measurements made at each sample position with a NaI:Tl detector using the 'energy windows' method detailed in Arnold et al. (2012b). Radionuclide concentrations were converted to dose rates using published conversion factors (Guérin et al., 2011)

${ }^{\mathrm{f}}$ Cosmic-ray dose rates were calculated according to Prescott and Hutton (1994) and assigned a relative uncertainty of $\pm 10 \%$.

g Includes an internal dose rate of $0.03 \pm 0.01 \mathrm{~Gy} / \mathrm{ka}$ after Bowler et al. (2003).

${ }^{\mathrm{h}}$ Mean \pm total uncertainty (68\% confidence interval), calculated as the quadratic sum of the random and systematic uncertainties. Total uncertainty includes a systematic component of $\pm 2 \%$ associated with laboratory beta-source calibration.

Table 3. TT-OSL dose rate data, single-grain equivalent doses and final ages for luminescence samples PM16-4, PM16-6, PM16-5 and PM16-3. 


\begin{tabular}{|c|c|c|c|c|c|c|c|c|c|}
\hline \multirow{2}{*}{ Boundary } & \multirow{2}{*}{$\begin{array}{l}\text { Dating } \\
\text { sample }\end{array}$} & \multicolumn{3}{|c|}{ Unmodelled age (years) $^{a}$} & \multicolumn{3}{|c|}{ Modelled age (years) } & \multirow{2}{*}{$\begin{array}{c}\text { Agreement } \\
\text { index } \\
\left(A_{i}\right) \\
(\%)\end{array}$} & \multirow{2}{*}{$\begin{array}{c}\text { Posterior outlier } \\
\text { probability } \\
(\%)\end{array}$} \\
\hline & & $68.2 \%$ range & $95.4 \%$ range & $\operatorname{Mean} \pm 1 \sigma$ & $68.2 \%$ range & 95.4\% range & $\operatorname{Mean} \pm 1 \sigma$ & & \\
\hline \multirow[t]{2}{*}{ Unit PM5 top } & & & & & $17400-7750$ & $17600-1050$ & $10700 \pm 4950$ & & \\
\hline & $\begin{array}{c}\text { pIR+OSL+TT-OSL } \\
\text { PM16-4 }{ }^{\text {b }}\end{array}$ & $17750-15900$ & $18600-15050$ & $16850 \pm 900$ & $17750-15900$ & $18650-15000$ & $16850 \pm 950$ & 100.2 & 5 \\
\hline \multirow[t]{2}{*}{ Unit PM5 bottom } & & & & & $29950-15900$ & $47900-15250$ & $26850 \pm 9900$ & & \\
\hline & ESR MIN1401 & $57800-37250$ & $67600-27400$ & $47500 \pm 10050$ & $58650-39100$ & $68600-29550$ & $49000 \pm 9700$ & 101.1 & 0 \\
\hline \multirow[t]{4}{*}{ Unit PM4 top } & & & & & $223400-179300$ & $236550-111750$ & $188550 \pm 35150$ & & \\
\hline & ESR MIN1402 & $225900-185150$ & $245450-165600$ & $205500 \pm 19950$ & $223500-195150$ & $234500-176550$ & $206900 \pm 14600$ & 118.0 & 3 \\
\hline & pIR+TT-OSL PM16-6 ${ }^{\mathrm{b}}$ & $236150-206200$ & $250550-191850$ & $221200 \pm 14700$ & $228750-207050$ & $238900-195450$ & $217350 \pm 10750$ & 116.0 & 3 \\
\hline & ESR VI1206 & $259300-216250$ & $280000-195550$ & $237800 \pm 21100$ & $237200-213950$ & $249000-202350$ & $225600 \pm 11500$ & 110.5 & 5 \\
\hline Unit PM4 bottom & & & & & $245900-218900$ & $259750-206900$ & $232750 \pm 13500$ & & \\
\hline \multirow[t]{5}{*}{ Unit PM3 top } & & & & & $263450-239250$ & $274600-226600$ & $250950 \pm 12150$ & & \\
\hline & pIR+TT-OSL PM16-5 b & $275400-232400$ & $296050-211700$ & $253900 \pm 21100$ & $265650-242650$ & $276400-231900$ & $254100 \pm 11100$ & 128.6 & 2 \\
\hline & ESR VI1204 & $305500-252200$ & $331050-226600$ & $278850 \pm 26100$ & $267600-246450$ & $278550-235750$ & $257150 \pm 10750$ & 96.8 & 7 \\
\hline & ESR VI1205 & $288850-242200$ & $311300-219750$ & $265500 \pm 22900$ & $270900-248500$ & $281900-238000$ & $259900 \pm 10950$ & 126.9 & 3 \\
\hline & pIR+TT-OSL PM16-3 ${ }^{b}$ & $276100-226900$ & $299700-203250$ & $251500 \pm 24100$ & $274000-250150$ & $286300-239300$ & $262550 \pm 11550$ & 121.1 & 2 \\
\hline Unit PM3 bottom & & & & & $276900-251950$ & $291600-241050$ & $265650 \pm 12900$ & & \\
\hline \multirow[t]{2}{*}{ Unit PM1-2 top } & & & & & $296500-262850$ & $317800-249650$ & $282350 \pm 17400$ & & \\
\hline & ESR MIN1403 & $291650-236950$ & $317950-210650$ & $264300 \pm 26800$ & $311650-274350$ & $332800-260150$ & $295250 \pm 18400$ & 78.4 & 1 \\
\hline Unit PM1-2 bottom & & & & & $424000-283800$ & $424000-280700$ & $342650 \pm 43500$ & & \\
\hline
\end{tabular}

a The original dating results are all expressed in years before AD2014 (average sample collection year) for modelling purposes to ensure the likelihoods are directly comparable.

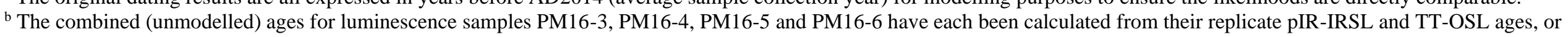
replicate pIR-IRSL, OSL and TT-OSL ages, using the OxCal Combine function. See Supplementary Information text for further details.

Table 4. Summary of Bayesian modelling results for Porto Maior (Model 1; see main text). The likelihood (unmodelled) and posterior (modelled) age ranges are presented for each of the numerical dating samples. Posterior (modelled) age ranges are also shown for the boundaries of each stratigraphic unit. Posterior ages are presented as the $68.2 \%$ and $95.4 \%$ highest probability density ranges. The mean and $1 \sigma$ uncertainty ranges of the modelled posterior distributions are shown for comparison (assuming a normally distributed probability density function). The unmodelled and modelled age estimates have been rounded to the nearest 50 years. 


\begin{tabular}{|c|c|c|c|c|c|c|c|}
\hline \multirow{2}{*}{ Unit / boundary } & \multirow{2}{*}{ Time variable } & \multicolumn{3}{|c|}{ Modelled age (years) ${ }^{a}$} & \multicolumn{3}{|c|}{ Modelled duration (years) ${ }^{a, b}$} \\
\hline & & $68.2 \%$ range & $95.4 \%$ range & Mean $\pm 1 \sigma$ & $68.2 \%$ range & $95.4 \%$ range & Mean $\pm 1 \sigma$ \\
\hline PM5 & age & $23050-11350$ & $36100-3900$ & $18800 \pm 7650$ & & & \\
\hline PM5 & duration & & & & $-50-20450$ & $-50-39700$ & $16150 \pm 12050$ \\
\hline PM5 - PM4 boundary & duration & & & & $149500-200250$ & $82200-215400$ & $161650 \pm 36350$ \\
\hline PM4 & age & $234300-200300$ & $251050-159650$ & $210650 \pm 24650$ & & & \\
\hline PM4 & duration & & & & $-50-51250$ & $-50-127450$ & $44200 \pm 39900$ \\
\hline PM4 - PM3 boundary & duration & & & & $-50-23100$ & $-50-43900$ & $18250 \pm 13300$ \\
\hline PM3 & age & $269900-246150$ & $282300-234200$ & $258300 \pm 12100$ & & & \\
\hline PM3 & duration & & & & $-50-17600$ & $-50-41750$ & $14700 \pm 13050$ \\
\hline PM3 - PM2 boundary & duration & & & & $-50-20550$ & $-50-4600$ & $16700 \pm 14350$ \\
\hline PM1-2 & age & $330050-272250$ & $382750-259650$ & $312550 \pm 32600$ & & & \\
\hline PM1-2 & duration & & & & $-50-81300$ & $-50-141500$ & $60300 \pm 45200$ \\
\hline
\end{tabular}

${ }^{a}$ Modelled age ranges were calculated from the posterior probabilities of the upper and lower boundaries (top and bottom) of each stratigraphic unit (see Table 4) using the date query function in OxCal v4.3. Modelled durations were calculated using the difference query function, and provide the temporal range between the posterior probability density distributions of successive stratigraphic boundaries.

b The OxCal difference function can be used to test whether or not the posterior probability distributions of successive stratigraphic boundaries are significantly different from each other at a given confidence interval. When the difference function is applied to adjacent boundaries of two different stratigraphic units (e.g. LU-6 - LU-5 boundary), it provides a statistical indication of the presence or absence of potential depositional hiatuses (given the available dating evidence). Calculated duration ranges that overlap with 0 at the $95.4 \%$ confidence interval suggest that the boundaries of successive units are not separated by a statistically significant temporal hiatus. Calculated duration ranges that do not overlap with 0 at the $95.4 \%$ confidence interval indicate potentially missing material and / or a temporal gap between the boundaries of successive units. When the difference function is applied to the upper and lower boundaries of the same stratigraphic unit (as opposed to the adjacent boundaries of successive units), a calculated duration of $>0$ years indicate a statistically significant temporal difference between the onset and termination of the depositional event. In contrast, calculated duration ranges that overlap with 0 indicate statistically indistinguishable onset and termination ages for the depositional event at a given confidence interval.

Table 5. Bayesian modelled posterior age ranges and depositional durations for the Porto Maior stratigraphic units (Model 1). Posterior ages/durations are presented as the $68.2 \%$ and $95.4 \%$ highest probability density ranges. The mean and $1 \sigma$ uncertainty ranges of the modelled posterior distributions are shown for comparison (assuming a normally distributed probability density function). The unmodelled and modelled age estimates have been rounded to the nearest 50 years. 


\begin{tabular}{|c|c|c|c|c|c|c|c|c|c|c|c|c|c|}
\hline \multirow[b]{2}{*}{ Boundary } & \multirow[b]{2}{*}{ Model } & \multicolumn{4}{|c|}{ Modelled age ${ }^{a}$} & \multicolumn{4}{|c|}{ Difference in modelling results (Model 2 - Model 1) } & \multicolumn{4}{|c|}{ Paired-value homogeneity test a } \\
\hline & & $\begin{array}{c}\text { 68.2\% range } \\
\quad(\text { years })\end{array}$ & $\begin{array}{l}95.4 \% \text { range } \\
\text { (years) }\end{array}$ & $\begin{array}{l}\text { Mean } \pm 1 \sigma \\
\quad(\text { years })\end{array}$ & $\begin{array}{c}\begin{array}{c}\text { Relative } \\
\text { uncertainty } \\
( \pm 1 \sigma)(\%)\end{array} \\
\end{array}$ & $\begin{array}{c}\Delta \text { mean age } \\
(\text { years })\end{array}$ & $\begin{array}{c}\% \text { change } \\
\text { in mean age } \\
(\%)\end{array}$ & $\begin{array}{c}\Delta \text { mean age } \\
\text { uncertainty } \\
( \pm 1 \sigma) \text { (years) } \\
\end{array}$ & $\begin{array}{c}\Delta \text { relative } \\
\text { uncertainty } \\
( \pm 1 \sigma)(\%) \\
\end{array}$ & G-value & $\begin{array}{c}\text { G-value } \\
<\mathbf{X}^{2} \text { crit }(\alpha: 0.05)\end{array}$ & $\begin{array}{c}\text { G-value } \\
<\mathrm{X}^{2} \text { crit }(\alpha: 0.32)\end{array}$ & P-value \\
\hline \multirow[t]{2}{*}{ Unit PM5 top } & 1 & $17400-7750$ & $17600-1050$ & $10700 \pm 4950$ & 46 & & & & & & & & \\
\hline & 2 & $17450-10400$ & $17600-1850$ & $12050 \pm 4750$ & 39 & 1350 & 13 & -200 & -7 & 0.04 & Yes & Yes & 0.84 \\
\hline \multirow[t]{2}{*}{ Unit PM5 bottom } & 1 & $29950-15900$ & $47900-15250$ & $26850 \pm 9900$ & 37 & & & & & & & & \\
\hline & 2 & $25200-16000$ & $44950-15400$ & $24250 \pm 9150$ & 38 & -2600 & -10 & -750 & -1 & 0.04 & Yes & Yes & 0.85 \\
\hline \multirow[t]{2}{*}{ Unit PM4 top } & 1 & $223400-179300$ & $236550-111750$ & $188550 \pm 35150$ & 19 & & & & & & & & \\
\hline & 2 & $226300-180200$ & $234400-111300$ & $189300 \pm 36350$ & 19 & 750 & $<1$ & 1200 & 0 & $<0.01$ & Yes & Yes & 0.99 \\
\hline \multirow[t]{2}{*}{ Unit PM4 bottom } & 1 & $245900-218900$ & $259750-206900$ & $232750 \pm 13500$ & 6 & & & & & & & & \\
\hline & 2 & $255700-218800$ & $307100-206750$ & $238150 \pm 18350$ & 8 & 5400 & 2 & 4850 & 2 & 0.06 & Yes & Yes & 0.81 \\
\hline \multirow[t]{2}{*}{ Unit PM3 top } & 1 & $263450-239250$ & $274600-226600$ & $250950 \pm 12150$ & 5 & & & & & & & & \\
\hline & 2 & $274500-238100$ & $290800-218150$ & $255200 \pm 18250$ & 7 & 4250 & 2 & 6100 & 2 & 0.04 & Yes & Yes & 0.85 \\
\hline \multirow[t]{2}{*}{ Unit PM3 bottom } & 1 & $276900-251950$ & $291600-241050$ & $265650 \pm 12900$ & 5 & & & & & & & & \\
\hline & 2 & $284400-247400$ & $304300-229600$ & $266500 \pm 18700$ & 7 & 850 & $<1$ & 5800 & 2 & $<0.01$ & Yes & Yes & 0.97 \\
\hline \multirow[t]{2}{*}{ Unit PM1-2 top } & 1 & $296500-262850$ & $317800-249650$ & $282350 \pm 17400$ & 6 & & & & & & & & \\
\hline & 2 & $296300-255400$ & $320450-237300$ & $277700 \pm 20850$ & 8 & -4650 & -2 & 3450 & 2 & 0.03 & Yes & Yes & 0.86 \\
\hline \multirow[t]{3}{*}{ Unit PM1-2 bottom } & 1 & $424000-283800$ & $424000-280700$ & $342650 \pm 43500$ & 13 & & & & & & & & \\
\hline & 2 & $427050-269700$ & $435450-259350$ & $330700 \pm 49400$ & 15 & -11950 & -4 & 5900 & 2 & 0.03 & Yes & Yes & 0.86 \\
\hline & & & & & Average & -800 & 0.3 & 3300 & 1 & & & & \\
\hline
\end{tabular}

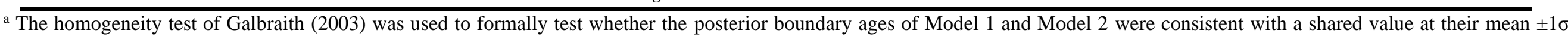

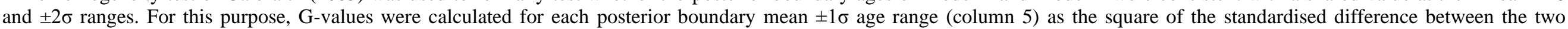

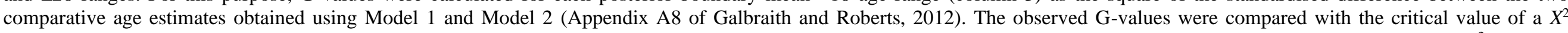

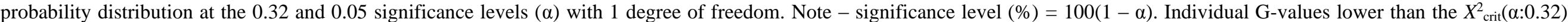

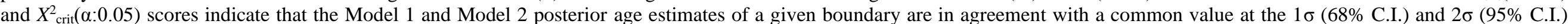

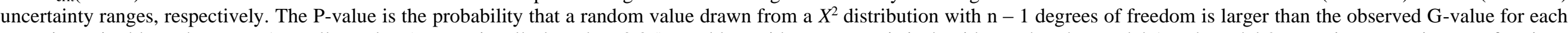

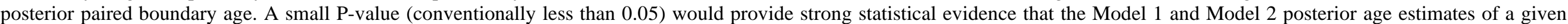
boundary are not consistent with a common value (Galbraith and Roberts, 2012).

Table 6. Comparison of the Bayesian modelled posterior age ranges for the upper and lower boundaries (top and bottom) of each stratigraphic unit obtained using Model 1 and Model 2. Model 1 was run using the combined (random + systematic) empirical dating uncertainties of each luminescence and ESR sample as likelihood input parameters. Model 2 was run after excluding all shared systematic error terms from each luminescence and ESR likelihood input parameter. For the model 2 results shown here, the average shared systematic uncertainty of the 15 likelihood (unmodelled) ages has subsequently been added in quadrature 
to the posterior (modelled) probabilities of the upper and lower boundaries of each unit to enable direct comparisons with the posterior (modelled) probability ranges of Model 1 . The modelled age estimates have been rounded to the nearest 50 years. 


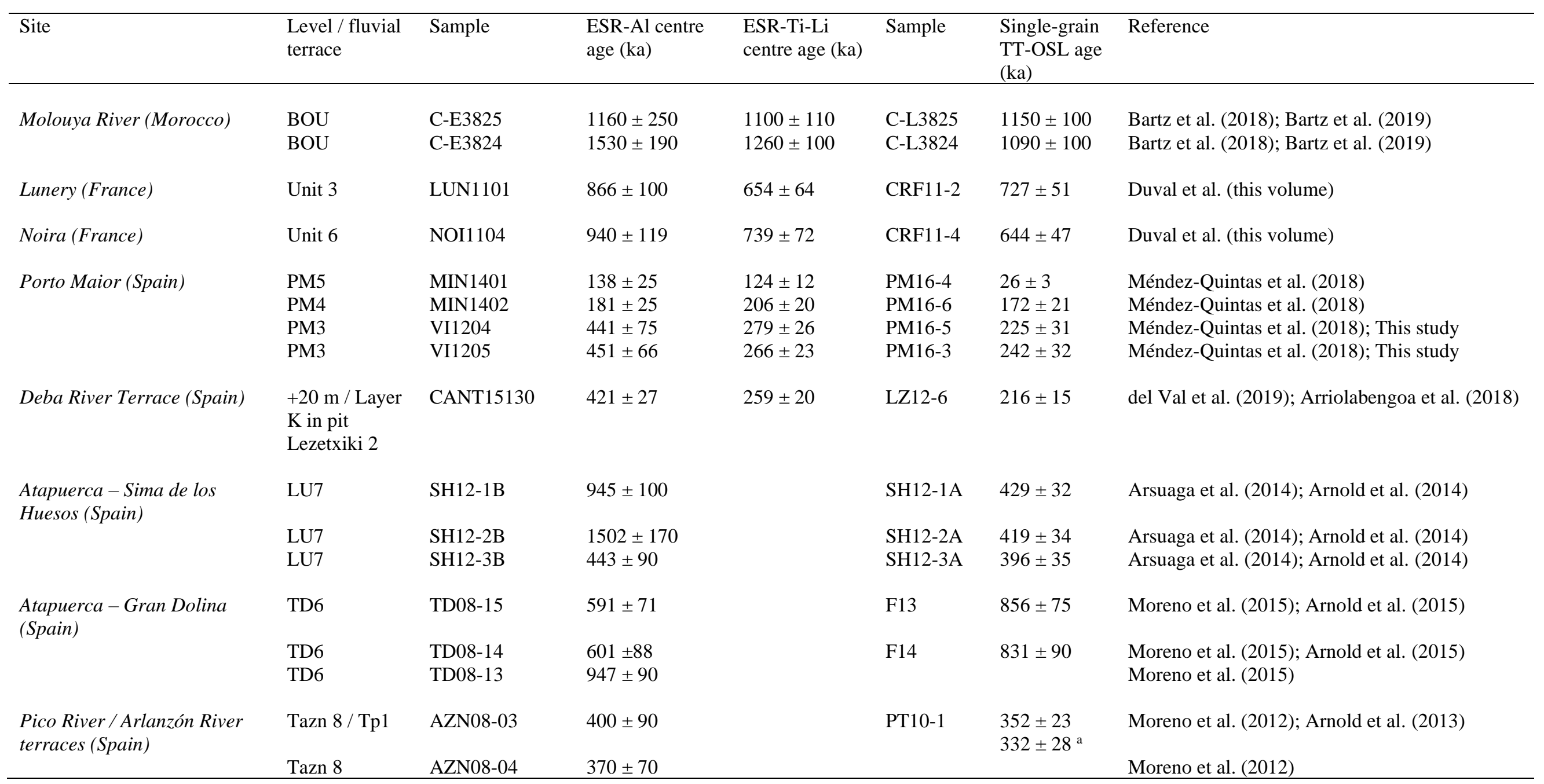

${ }^{a}$ This age was derived from measurements made on multi-grain aliquots.

Table 7. Comparative chronologies obtained using single-grain TT-OSL and ESR dating (based on the Al and Ti-Li centres) of optically-bleached quartz from a range of archaeological sites and fluvial deposits from Spain, France and Morocco. 


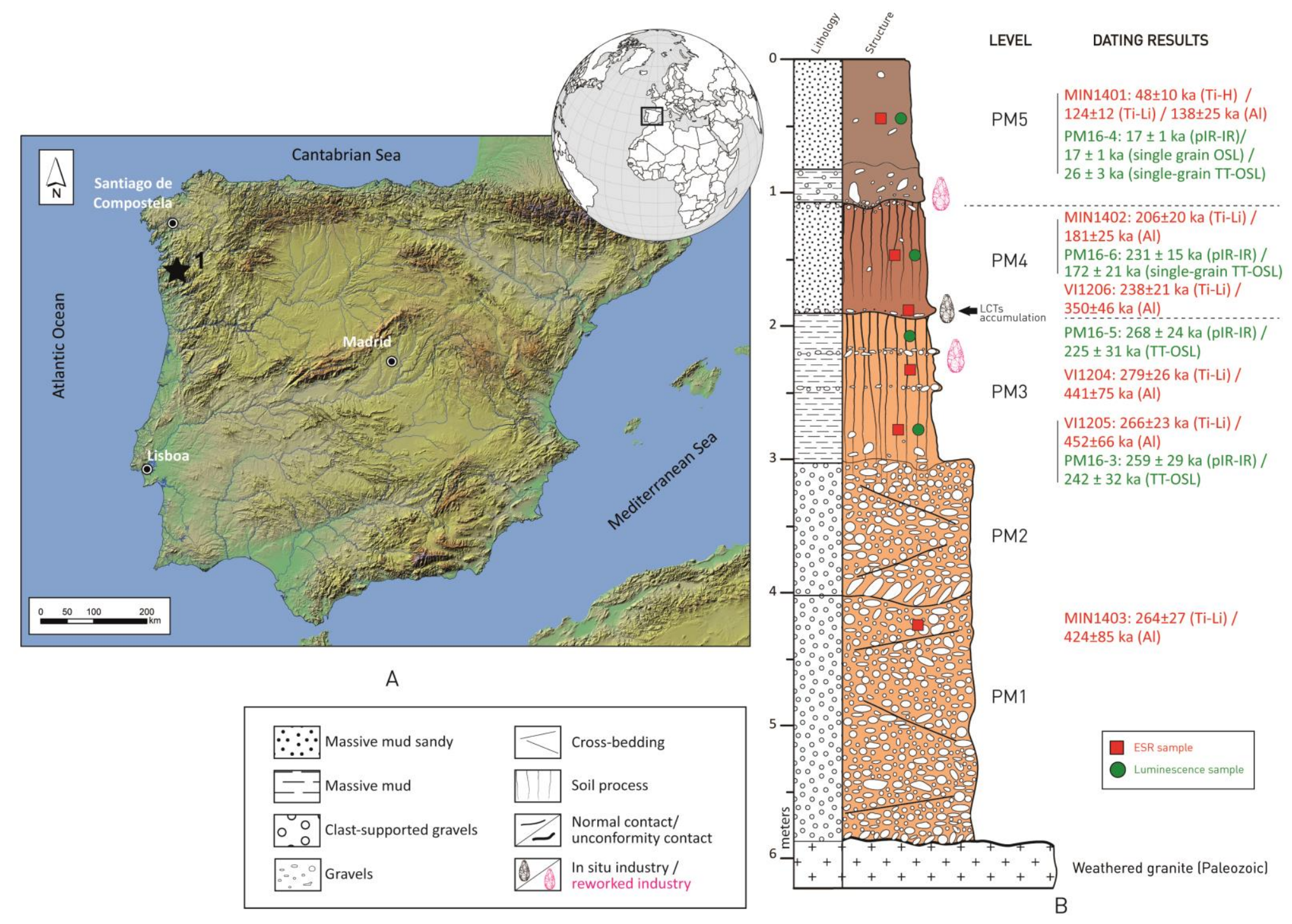

Figure 1. (A) Location of Porto Maior (black star), Galicia, Spain. (B) Stratigraphic section showing the previously published pIR-IRSL, single-grain OSL and ESR ages for Porto Maior, together with the new TT-OSL ages obtained in the present study (modified from Méndez-Quintas et al., 2018). 
A

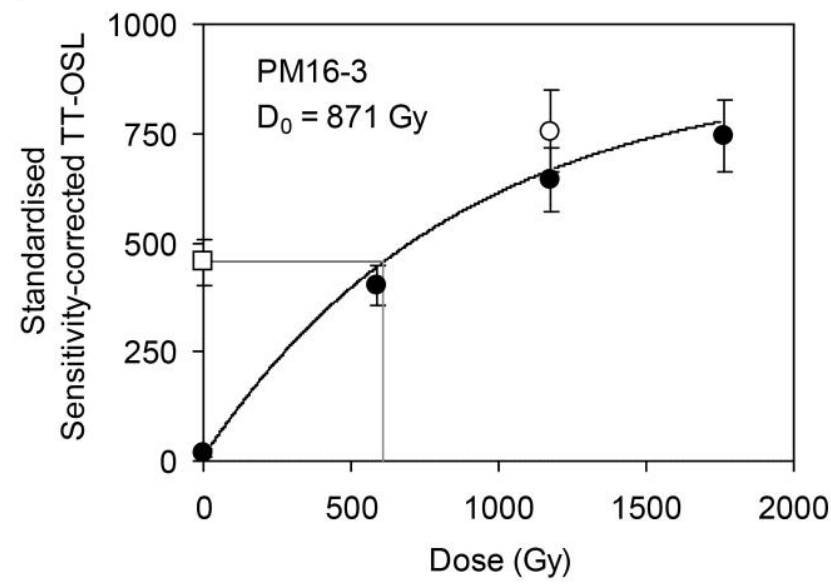

C

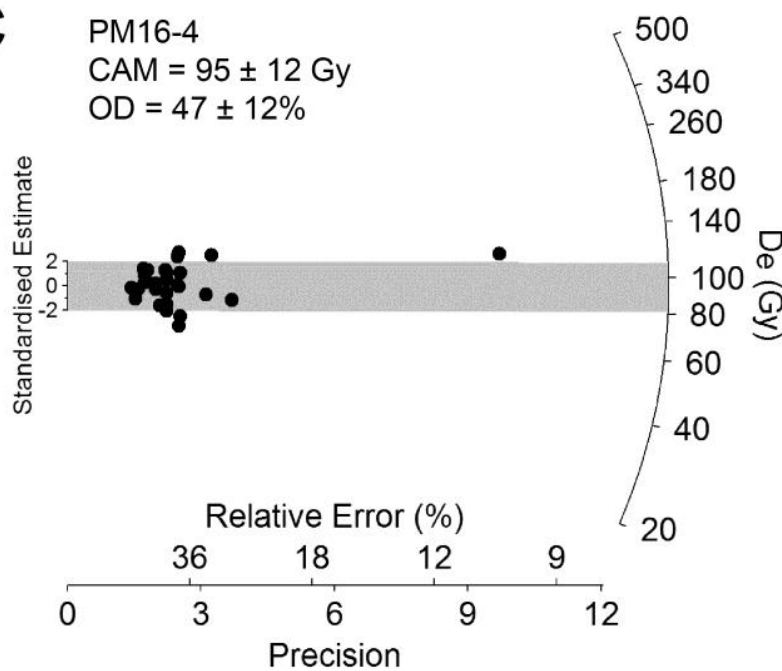

$E$

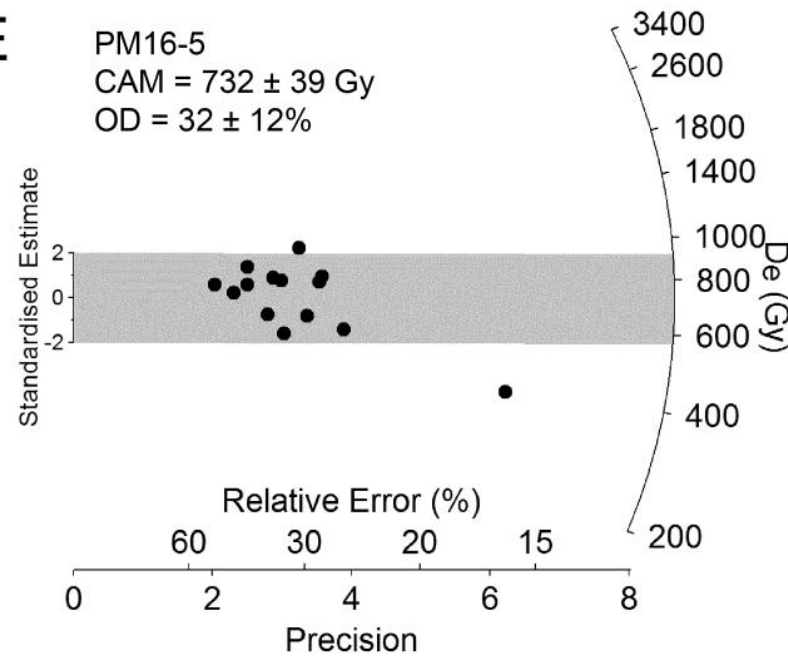

B
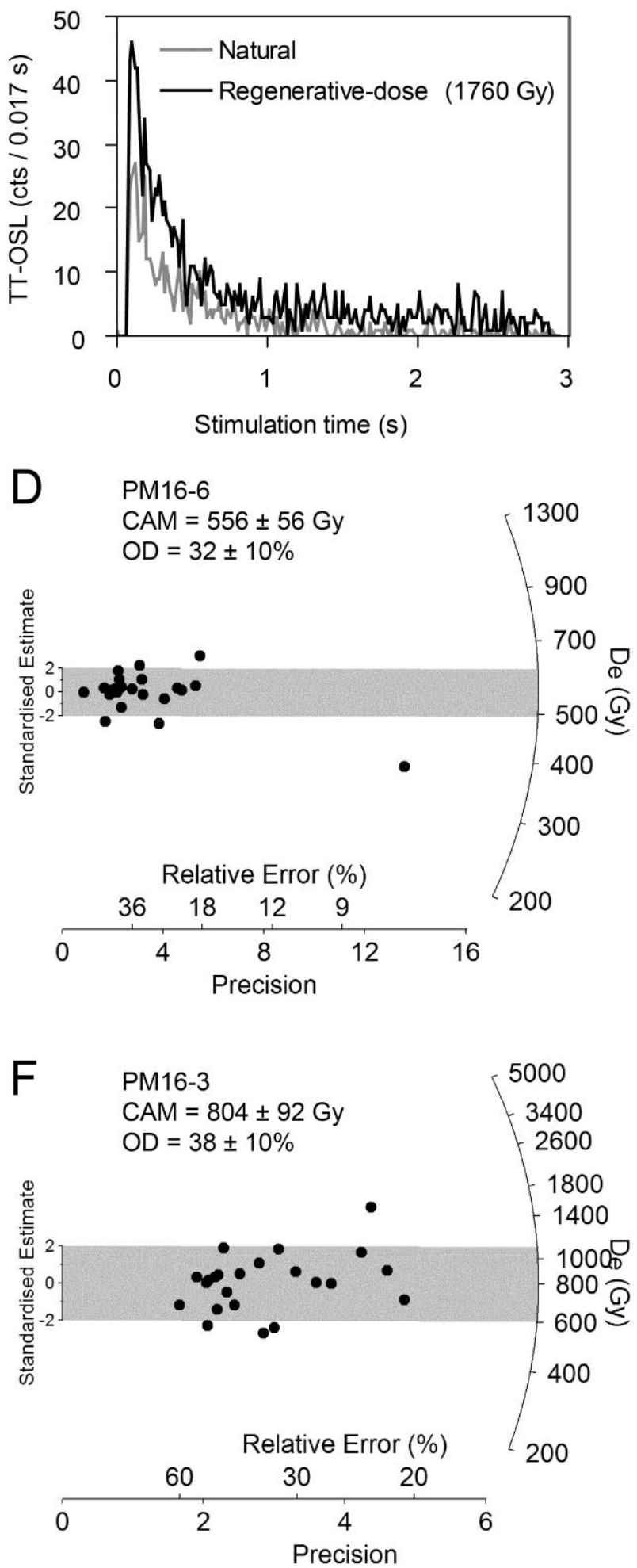

Figure 2. (A, B) Example of a sensitivity-corrected TT-OSL dose-response and decay curve for an individual grain from sample PM16-3. White square denotes the sensitivity-corrected natural OSL signal; filled circles denote the sensitivity-corrected regenerated OSL signals; white circle denotes the repeated regenerative dose point used to calculate the recycling ratio. The $\mathrm{D}_{0}$ value characterises the rate of signal saturation with respect to administered dose and equates to the dose value for which the saturating exponential dose-response curve slope is $1 / e$ (or $~ 0.37$ ) of its initial value. $(\mathrm{C}-\mathrm{F})$ The single-grain TTOSL $D_{\mathrm{e}}$ distributions obtained for samples PM16-4, PM16-6, PM16-5 and PM16-3. The grey bars on the radial plots are centred on the central age model $(\mathrm{CAM}) \mathrm{D}_{\mathrm{e}}$ value used to derive the final burial dose estimate for each sample. Also shown are the overdispersion (OD) values associated with each $\mathrm{D}_{\mathrm{e}}$ dataset. 


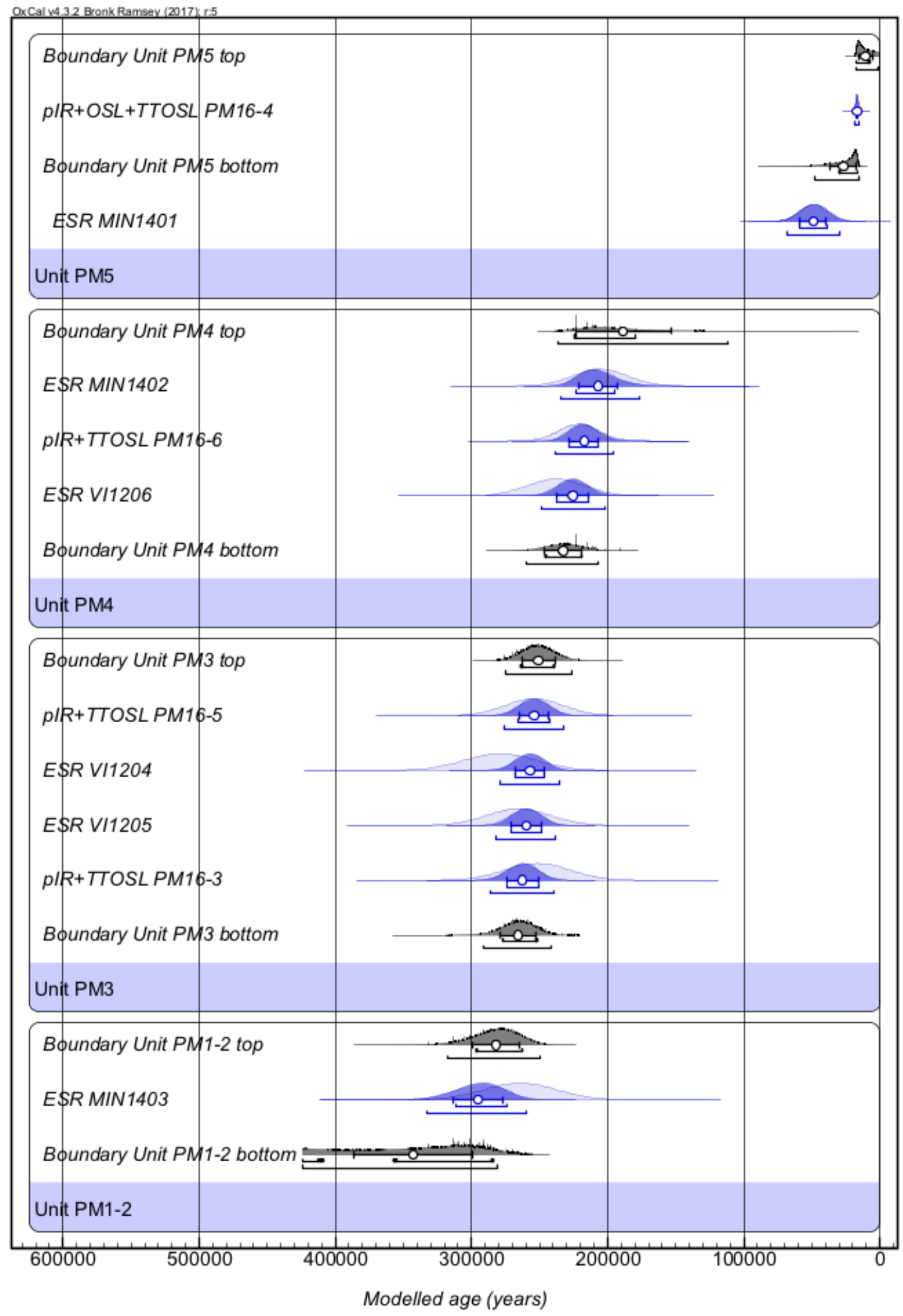

Figure 3. Bayesian modelling results for the Porto Maior stratigraphic sequence (Model 1; see main text). The prior age distributions for the dating samples (likelihoods) are shown as light blue probability density functions (PDFs). The modelled posterior distributions for the dating samples and stratigraphic unit boundaries are shown as dark blue and grey PDFs, respectively. Unmodelled and modelled ages are shown on a calendar year timescale and both are expressed in years before AD2014 (average sample 
collection date). The white circles and associated error bars represent the mean ages and $1 \sigma$ uncertainty ranges of the PDFs. The $68.2 \%$ and $95.4 \%$ ranges of the highest posterior probabilities are indicated by the horizontal bars underneath the PDFs. 


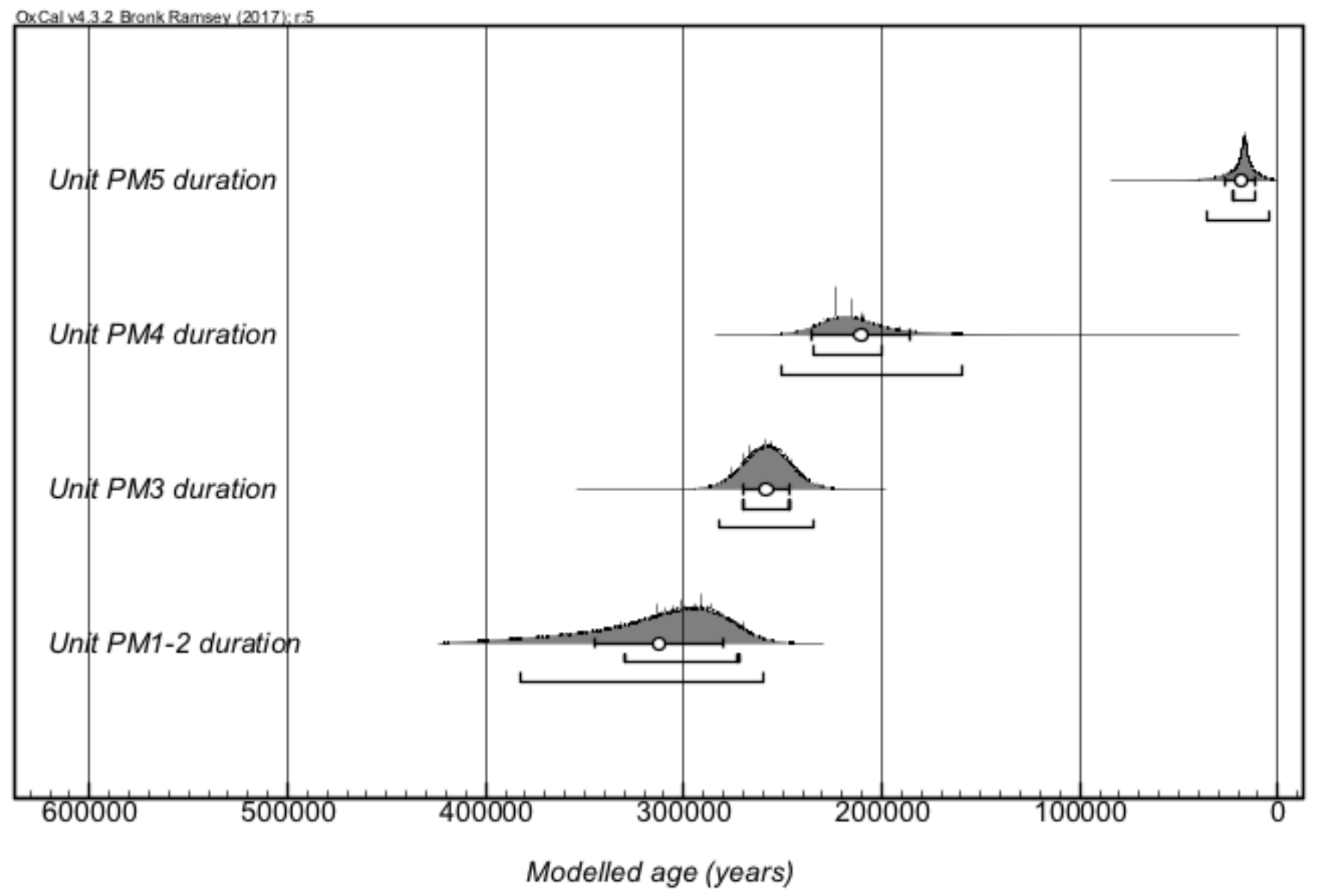

Figure 4. Bayesian-modelled durations of the stratigraphic units at Porto Maior (Model 1; see main text). The PDFs have been calculated from the modelled posterior probabilities of the upper and lower boundaries of each stratigraphic unit (shown as grey PDFs in Figure 5) using the date query function in OxCal v4.3.2. 
A

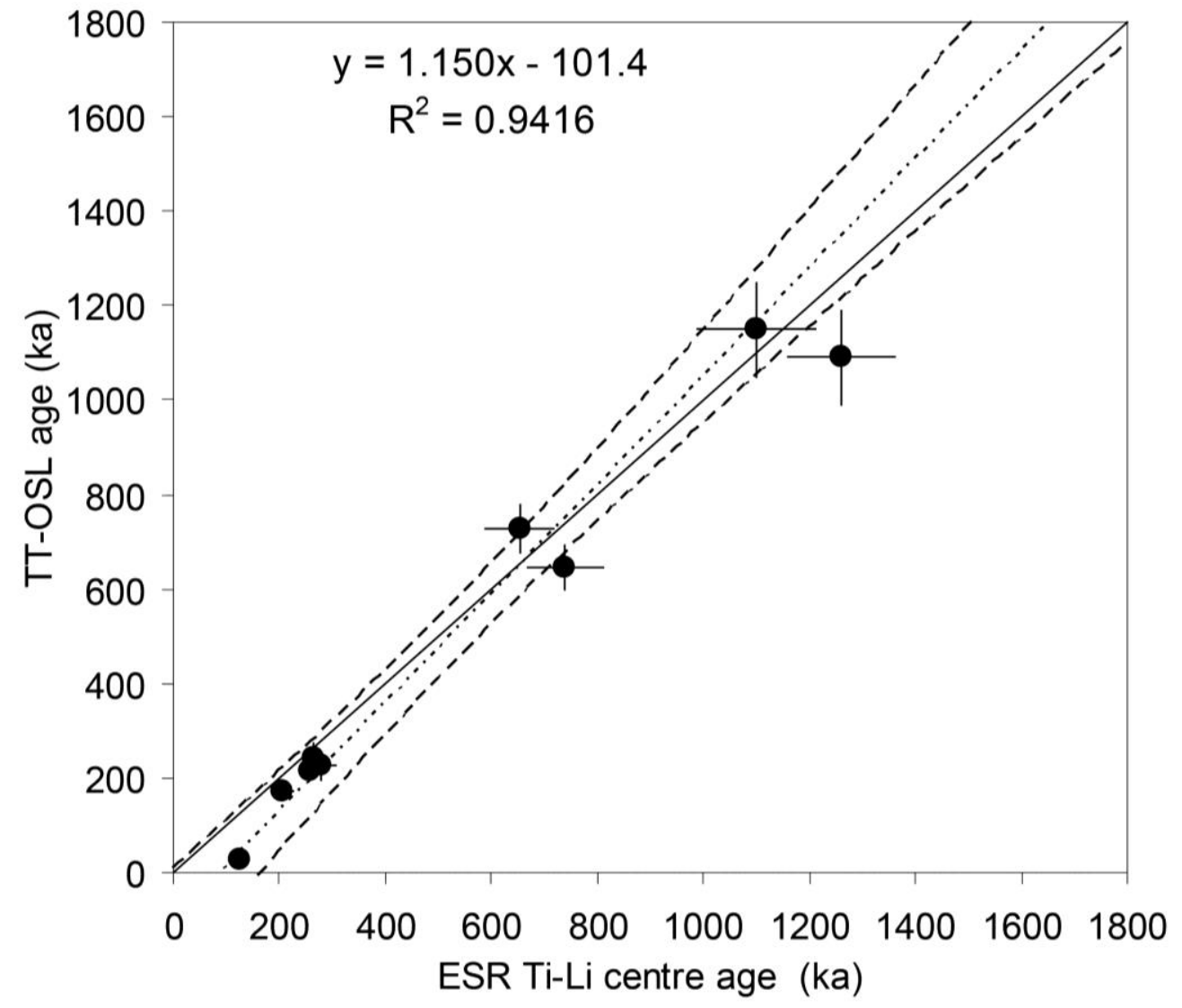

B

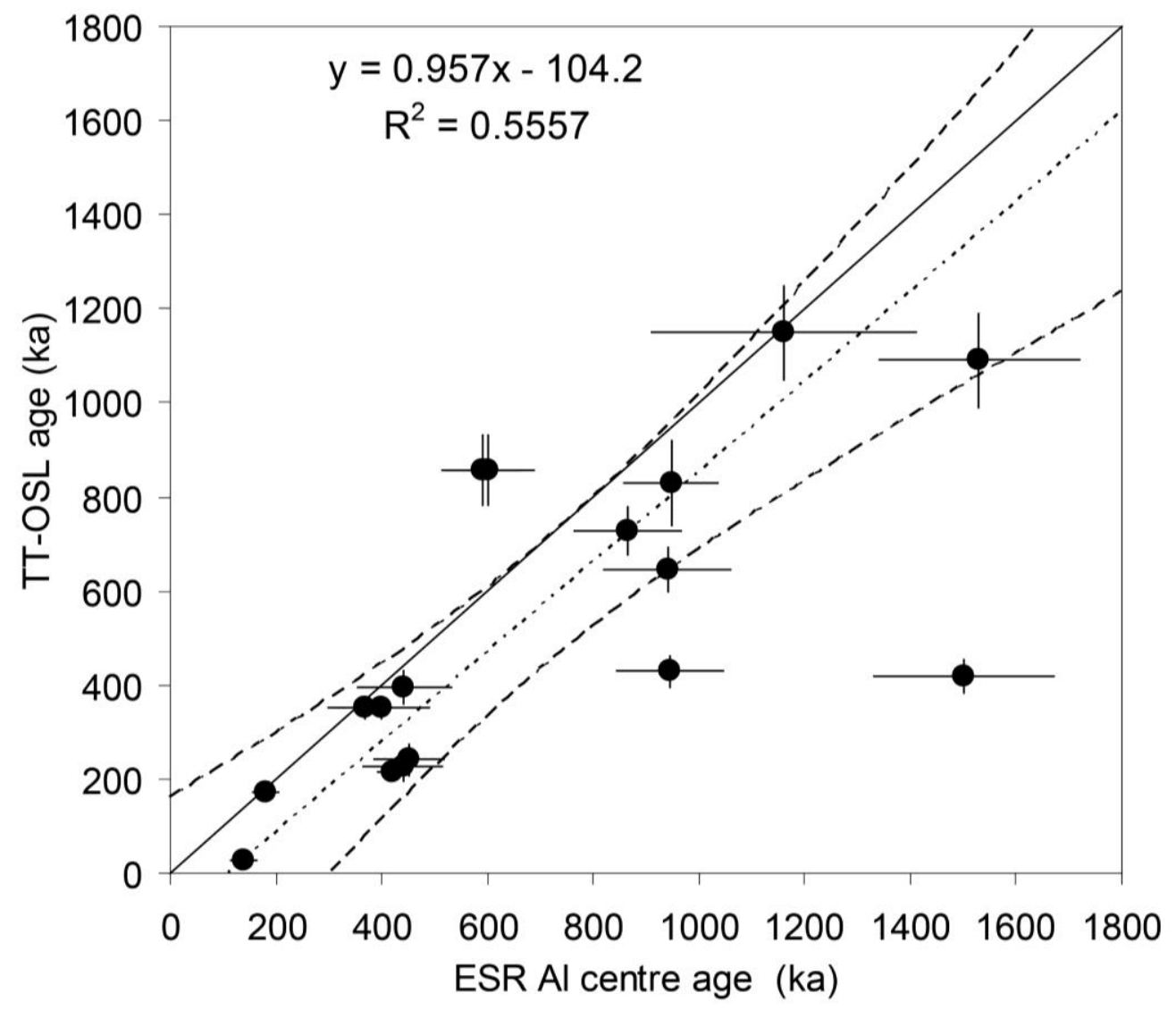

Figure 5. ESR and TT-OSL age comparisons based on published dating studies conducted on a range of sites from Spain, France and Morocco (based on data shown in Table 7). The uncertainties for each age estimate represent the $1 \sigma$ errors. The type of ESR centre used to derive comparative ages for each TTOSL sample correspond to the Ti-Li centre (option D) in plot (A) and the Al centre in plot (B). Each dataset has been fitted with a linear least-squares function (dashed line) weighted according to the inverse squared relative errors of both coordinates, following the approach of Reed (1989). The confidence intervals about the linear regression functions (long-dashed lines) have been calculated using the Student's t-statistic for a two-tailed test with (n-2) degrees of freedom and are presented at the $95.4 \%$ confidence level $(\alpha=0.05)$. The solid line represents the $1: 1$ line for each dataset. 
Supplementary Information - Demuro et al. Refining the chronology of Acheulean deposits at Porto Maior in the River Miño basin (Galicia, Spain) using a comparative luminescence and ESR dating approach.

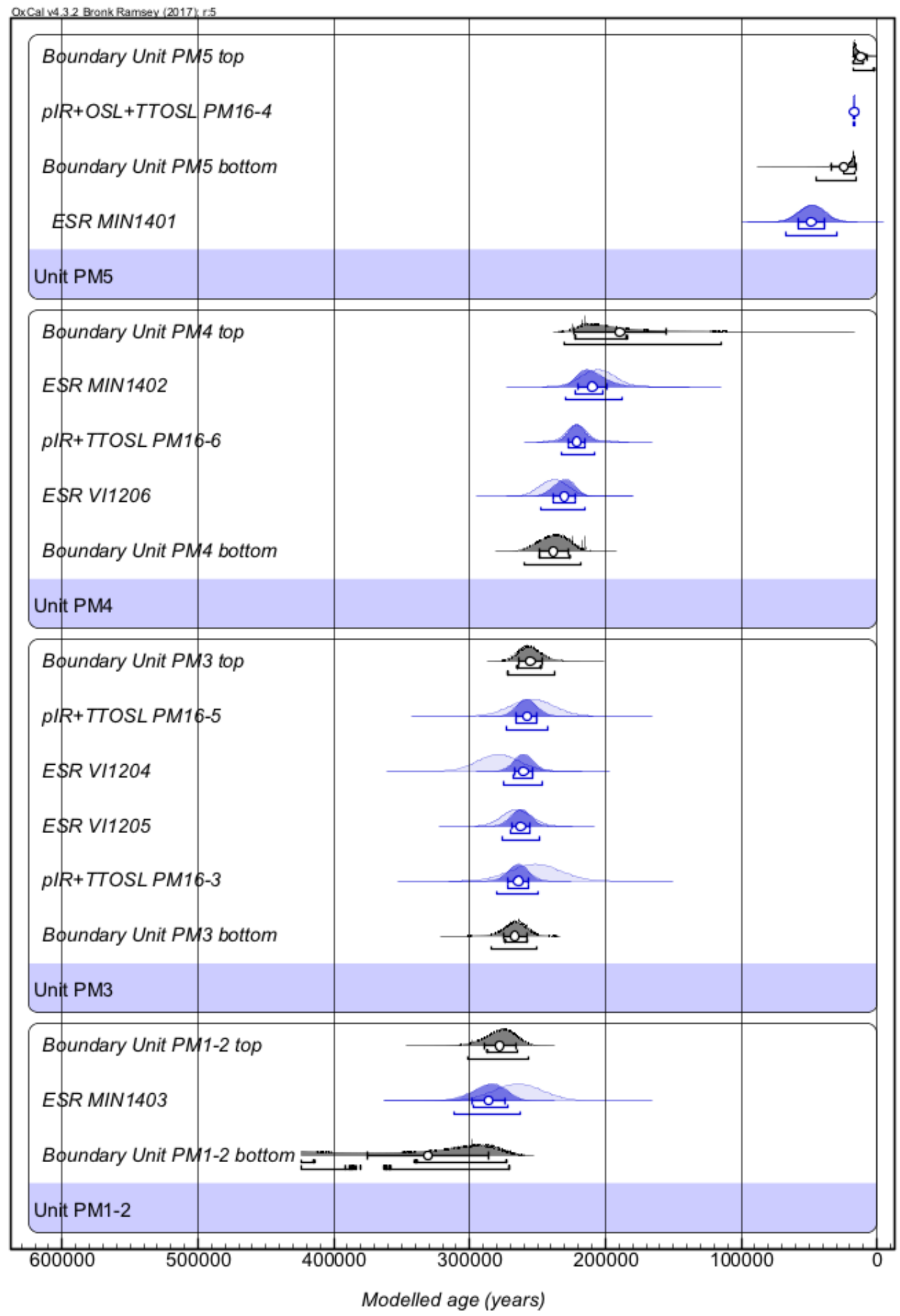

Figure S1 - Bayesian modelling results for the Porto Maior stratigraphic sequence (Model 2; see main text). The prior age distributions for the dating samples (likelihoods) are shown as light blue probability density functions (PDFs). The modelled posterior distributions for the dating samples and stratigraphic unit boundaries are shown as dark blue and grey PDFs, respectively. Unmodelled and modelled ages are shown on a calendar year timescale and both are expressed in years before AD2014 (average sample collection date). The white circles and associated error bars represent the mean ages and $1 \sigma$ uncertainty ranges of the PDFs. The $68.2 \%$ and 95.4\% ranges of the highest posterior probabilities are indicated by the horizontal bars underneath the PDFs. 


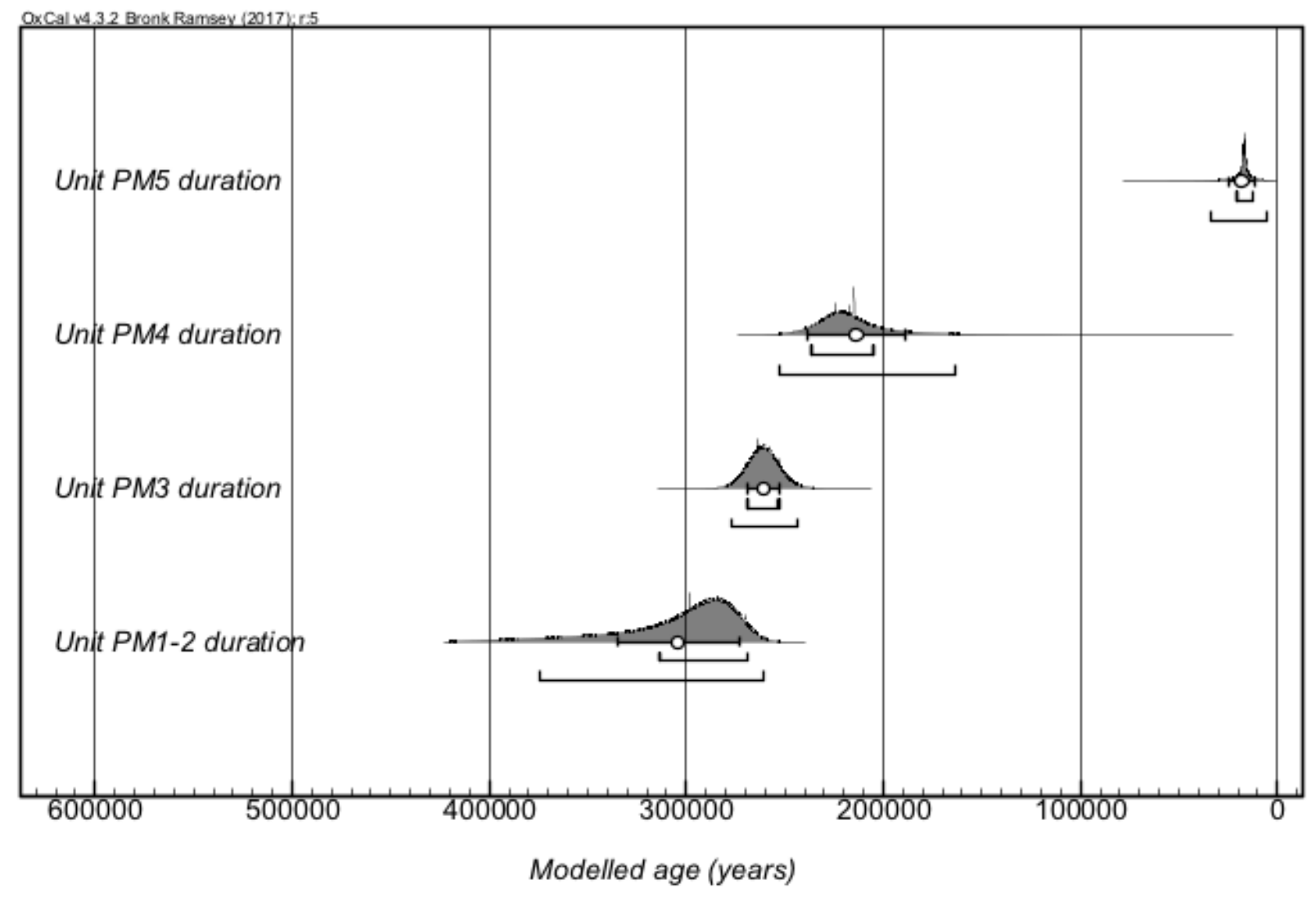

Figure S2 - Bayesian-modelled durations of the stratigraphic units at Porto Maior (Model 2; see main text). The PDFs have been calculated from the modelled posterior probabilities of the upper and lower boundaries of each stratigraphic unit (shown as grey PDFs in Figure S1) using the date query function in OxCal v4.3.2. 
Natural dose assessment (un-dosed grains)

Dose recovery test

\begin{tabular}{|c|c|c|c|c|c|c|c|}
\hline Sample & Accepted / measured & $\mathrm{CAM} \mathrm{D}_{\mathrm{e}}(\mathrm{Gy})$ & Given dose (Gy) & Accepted / measured & $\mathrm{CAM}_{\mathrm{e}}(\mathrm{Gy})$ & $\mathrm{OD}(\%)$ & $\begin{array}{l}\text { Measured / given dose ratio } \\
\text { (after residual subtraction) }\end{array}$ \\
\hline PM16-3 & $23 / 1300$ & $804 \pm 92$ & $578 \pm 4$ & $18 / 500$ & $1368 \pm 117$ & $19 \pm 11$ & $0.98 \pm 0.26$ \\
\hline
\end{tabular}

${ }^{a}$ Mean \pm total uncertainty ( $68 \%$ confidence interval), calculated as the quadratic sum of the random and systematic uncertainties.

Table S1. Single-grain TT-OSL dose recovery test results obtained for sample PM16-3 using the SAR protocol shown in Table 2. 


\begin{tabular}{|c|c|c|c|c|c|c|c|c|}
\hline Sample name & PM16-4 & & PM16-6 & & PM16-5 & & PM16-3 & \\
\hline Total number of grains measured & 2600 & & 1300 & & 1000 & & 1300 & \\
\hline & $\mathrm{n}$ & $\%$ & $\mathrm{n}$ & $\%$ & $\mathrm{n}$ & $\%$ & $\mathrm{n}$ & $\%$ \\
\hline \multicolumn{9}{|l|}{ Reason for rejecting grains from $\mathrm{D}_{\mathrm{e}}$ analysis: } \\
\hline (i) $\mathrm{T}_{\mathrm{n}}<3 \sigma$ background (non-luminescent grains) & 1924 & 74.0 & 994 & 76.5 & 805 & 80.5 & 1009 & 77.6 \\
\hline (ii) Poor dose recycling ratio & 228 & 8.8 & 147 & 11.3 & 62 & 6.2 & 76 & 5.8 \\
\hline (iii) Recuperation ( 0 Gy $\mathrm{L}_{\mathrm{x}} / \mathrm{T}_{\mathrm{x}}>5 \%$ of $\left.\mathrm{L}_{\mathrm{n}} / \mathrm{T}_{\mathrm{n}}\right)$ & 25 & 1.0 & 0 & 0 & 0 & 0 & 5 & 0.4 \\
\hline (iv) Poor OSL IR depletion ratio & 7 & 0.3 & 0 & 0 & 2 & 0.2 & 1 & 0.1 \\
\hline (v) Relative error on the net $\mathrm{T}_{\mathrm{n}}$ signal $>30 \%$ & 387 & 14.9 & 133 & 10.2 & 114 & 11.4 & 176 & 13.5 \\
\hline $\begin{array}{l}\text { (vi) Saturated or non-intersecting grains }\left(\mathrm{L}_{\mathrm{n}} / \mathrm{T}_{\mathrm{n}} \approx \text { or }>\text { dose- }\right. \\
\text { response curve saturation) }\end{array}$ & 0 & 0 & 2 & 0.2 & 1 & 0.1 & 3 & 0.2 \\
\hline $\begin{array}{l}\text { (vii) Anomalous dose-response / unable to perform Monte } \\
\text { Carlo fit }{ }^{a}\end{array}$ & 2 & 0.1 & 2 & 0.2 & 2 & 0.2 & 7 & 0.5 \\
\hline Accepted grains (used for $D_{e}$ determination) & 27 & 1.0 & 22 & 1.7 & 14 & 1.4 & 23 & 1.8 \\
\hline
\end{tabular}

Includes grains with zero or negative changes in $\mathrm{L}_{x} / \mathrm{T}_{\mathrm{x}}$ with increasing dose, and grains displaying very scattered $\mathrm{L}_{\mathrm{x}} / \mathrm{T}_{\mathrm{x}}$ values that could not be successfully fitted with the Monte Carlo procedure.

Table S2. Single-grain TT-OSL quality assurance statistics obtained for natural De measurements of samples PM16-4, PM16-6, PM16-5 and PM16-3. 


\begin{tabular}{|c|c|c|c|c|c|c|c|c|c|}
\hline \multirow{2}{*}{ Boundary } & \multirow{2}{*}{$\begin{array}{l}\text { Dating } \\
\text { sample }\end{array}$} & \multicolumn{3}{|c|}{ Unmodelled age (years) ${ }^{a}$} & \multicolumn{3}{|c|}{ Modelled age (years) ${ }^{b}$} & \multirow{2}{*}{$\begin{array}{c}\text { Agreement } \\
\text { index } \\
\left(A_{i}\right) \\
(\%) \\
\end{array}$} & \multirow{2}{*}{$\begin{array}{c}\text { Posterior outlier } \\
\text { probability } \\
(\%)\end{array}$} \\
\hline & & 68.2\% range & 95.4\% range & $\operatorname{Mean} \pm 1 \sigma$ & 68.2\% range & 95.4\% range & $\operatorname{Mean} \pm 1 \sigma$ & & \\
\hline \multirow[t]{3}{*}{ Unit PM5 top } & & & & & $17350-10500$ & $17500-1950$ & $12050 \pm 4700$ & & \\
\hline & & & & & $(17450-10400)$ & $(17600-1850)$ & $(12050 \pm 4750)$ & & \\
\hline & $\begin{array}{c}\text { pIR+OSL+TT-OSL } \\
\text { PM16-4 }{ }^{\text {c }}\end{array}$ & $17250-16400$ & $17650-16000$ & $16850 \pm 400$ & $17250-16350$ & $17700-16000$ & $16850 \pm 450$ & 104.8 & 0 \\
\hline \multirow[t]{3}{*}{ Unit PM5 bottom } & & & & & $25000-16200$ & $44450-15900$ & $24250 \pm 9000$ & & \\
\hline & & & & & $(25200-16000)$ & $(44950-15400)$ & $(24250 \pm 9150)$ & & \\
\hline & ESR MIN1401 & $57200-37850$ & $66500-28550$ & $47500 \pm 9500$ & $58050-39250$ & $67250-30300$ & $48750 \pm 9200$ & 100.7 & 0 \\
\hline \multirow[t]{5}{*}{ Unit PM4 top } & & & & & $222500-184050$ & $230500-115200$ & $189300 \pm 34350$ & & \\
\hline & & & & & $(226300-180200)$ & $(234400-111300)$ & $(189300 \pm 36350)$ & & \\
\hline & ESR MIN1402 & $218050-193000$ & $230050-181000$ & $205500 \pm 12250$ & $221850-201950$ & $228950-187350$ & $209600 \pm 10700$ & 106.8 & 2 \\
\hline & pIR+TT-OSL PM16-6 ${ }^{c}$ & $228400-214000$ & $235350-207050$ & $221200 \pm 7050$ & $227300-215000$ & $232450-208450$ & $220950 \pm 6200$ & 112.6 & 1 \\
\hline & ESR VI1206 & $248500-227100$ & $258800-216800$ & $237800 \pm 10500$ & $238100-221850$ & $247150-215150$ & $230600 \pm 8050$ & 99.2 & 3 \\
\hline \multirow[t]{2}{*}{ Unit PM4 bottom } & & & & & $248200-226300$ & $259450-218400$ & $238150 \pm 10750$ & & \\
\hline & & & & & $(255700-218800)$ & $(307100-206750)$ & $(238150 \pm 18350)$ & & \\
\hline \multirow[t]{6}{*}{ Unit PM3 top } & & & & & $264900-247700$ & $271950-237000$ & $255200 \pm 8850$ & & \\
\hline & & & & & $(274500-238100)$ & $(290800-218150)$ & $(255200 \pm 18250)$ & & \\
\hline & pIR+TTOSL PM16-5 ${ }^{\mathrm{c}}$ & $270350-237400$ & $286200-221600$ & $253900 \pm 16150$ & $265900-250550$ & $273150-242000$ & $257750 \pm 7650$ & 129.5 & 1 \\
\hline & ESR VI1204 & $294100-263600$ & $308750-248900$ & $278850 \pm 14950$ & $267600-253400$ & $274450-246050$ & $260250 \pm 7050$ & 68.4 & 9 \\
\hline & ESR VI1205 & $276100-254950$ & $286300-244750$ & $265500 \pm 10400$ & $269350-255450$ & $276400-248300$ & $262350 \pm 6950$ & 118.5 & 2 \\
\hline & pIR+TTOSL PM16-3 ${ }^{c}$ & $270300-232700$ & $288350-214650$ & $251500 \pm 18450$ & $271650-256500$ & $279750-249150$ & $264250 \pm 7450$ & 111.1 & 1 \\
\hline \multirow[t]{2}{*}{ Unit PM3 bottom } & & & & & $274000-257800$ & $283750-250150$ & $266500 \pm 8450$ & & \\
\hline & & & & & $(284400-247400)$ & $(304300-229600)$ & $(266500 \pm 18700)$ & & \\
\hline \multirow[t]{3}{*}{ Unit PM1-2 top } & & & & & $286850-264800$ & $301550-256200$ & $277700 \pm 11550$ & & \\
\hline & & & & & $(296300-255400)$ & $(320450-237300)$ & $(277700 \pm 20850)$ & & \\
\hline & ESR MIN1403 & $282600-246000$ & $300200-228400$ & $264300 \pm 17950$ & $297050-272250$ & $311750-262550$ & $286200 \pm 12300$ & 74.9 & 1 \\
\hline \multirow[t]{2}{*}{ Unit PM1-2 bottom } & & & & & $424000-272750$ & $424000-270800$ & $330700 \pm 44850$ & & \\
\hline & & & & & $(427050-269700)$ & $(435450-259350)$ & $(330700 \pm 49400)$ & & \\
\hline
\end{tabular}

${ }^{a}$ The original dating results are all expressed in years before AD2014 (average sample collection year) for modelling purposes to ensure the likelihoods are directly comparable.

${ }^{\mathrm{b}}$ The modelled boundary age ranges and uncertainties shown outside parentheses represents the raw OxCal modelling output and are based on propagation of random dating uncertainties only. The modelled boundary age ranges and uncertainties shown in parentheses includes propagation of the modelled (random) dating uncertainties and the mean systematic uncertainty $(6.4 \%)$ of the thirteen numerical dating estimates in quadrature. 
Table S3. Summary of Bayesian modelling results for Porto Maior (Model 2; see main text). The likelihood (unmodelled) and posterior (modelled) age ranges are presented for each of the numerical dating samples. Posterior (modelled) age ranges are also shown for the boundaries of each stratigraphic unit. Posterior ages are presented as the $68.2 \%$ and $95.4 \%$ highest probability density ranges. The mean and $1 \sigma$ uncertainty ranges of the modelled posterior distributions are shown for comparison (assuming a normally distributed probability density function). The unmodelled and modelled age estimates have been rounded to the nearest 50 years. 


\begin{tabular}{|c|c|c|c|c|c|c|c|}
\hline \multirow{2}{*}{ Unit / boundary } & \multirow{2}{*}{ Time variable } & \multicolumn{3}{|c|}{ Modelled age (years) $^{a}$} & \multicolumn{3}{|c|}{ Modelled duration (years) ${ }^{a, b}$} \\
\hline & & $68.2 \%$ range & $95.4 \%$ range & Mean $\pm 1 \sigma$ & $68.2 \%$ range & $95.4 \%$ range & $\operatorname{Mean} \pm 1 \sigma$ \\
\hline PM5 & age & $24050-11050$ & $32950-6150$ & $18800 \pm 6900$ & & & \\
\hline PM5 & duration & & & & $3250-21050$ & $-150-30700$ & $14550 \pm 8650$ \\
\hline PM5 - PM4 boundary & duration & & & & $126650-193500$ & $89250-218950$ & $156300 \pm 3265$ \\
\hline PM4 & age & $241000-192100$ & $259800-157450$ & $211150 \pm 27250$ & & & \\
\hline PM4 & duration & & & & $15900-84200$ & $-450-120550$ & $57600 \pm 34050$ \\
\hline PM4 - PM3 boundary & duration & & & & $-14950-33150$ & $-39550-56100$ & $8500 \pm 23850$ \\
\hline PM3 & age & $276350-245750$ & $292000-230450$ & $260950 \pm 15850$ & & & \\
\hline PM3 & duration & & & & $-250-31850$ & $-250-62300$ & $25050 \pm 18600$ \\
\hline PM3 - PM2 boundary & duration & & & & $-23750-28550$ & $-50500-53600$ & $1850 \pm 25950$ \\
\hline PM1-2 & age & $332000-271300$ & $376800-251150$ & $309600 \pm 33700$ & & & \\
\hline PM1-2 & duration & & & & $9200-94650$ & $-3150-149450$ & $68550 \pm 42750$ \\
\hline
\end{tabular}

${ }^{\text {a }}$ Modelled age ranges were calculated from the posterior probabilities of the upper and lower boundaries (top and bottom) of each stratigraphic unit (see Table 5) using the date query function in OxCal v4.3. Modelled durations were calculated using the difference query function, and provide the temporal range between the posterior probability density distributions of successive stratigraphic boundaries.

$\mathrm{b}$ The OxCal difference function can be used to test whether or not the posterior probability distributions of successive stratigraphic boundaries are significantly different from each other at a given confidence interval. When the difference function is applied to adjacent boundaries of two different stratigraphic units (e.g. LU-6 - LU-5 boundary), it provides a statistical indication of the presence or absence of potential depositional hiatuses (given the available dating evidence). Calculated duration ranges that overlap with 0 at the $95.4 \%$ confidence interval suggest that the boundaries of successive units are not separated by a statistically significant temporal hiatus. Calculated duration ranges that do not overlap with 0 at the $95.4 \%$ confidence interval indicate potentially missing material and / or a temporal gap between the boundaries of successive units. When the difference function is applied to the upper and lower boundaries of the same stratigraphic unit (as opposed to the adjacent boundaries of successive units), a calculated duration of $>0$ years indicate a statistically significant temporal difference between the onset and termination of the depositional event. In contrast, calculated duration ranges that overlap with 0 indicate statistically indistinguishable onset and termination ages for the depositional event at a given confidence interval.

Table S4. Bayesian modelled posterior age ranges and depositional durations for the Porto Maior stratigraphic units (Model 2). Posterior ages/durations are presented as the $68.2 \%$ and $95.4 \%$ highest probability density ranges. The mean and $1 \sigma$ uncertainty ranges of the modelled posterior distributions are shown for comparison (assuming a normally distributed probability density function). For the model 2 results shown here, the average shared systematic uncertainty of the 15 likelihood (unmodelled) ages has subsequently been added in quadrature to the posterior (modelled) probabilities of the upper and lower boundaries of each unit to enable direct comparisons with the posterior (modelled) probability ranges of Model 1. The unmodelled and modelled age estimates have been rounded to the nearest 50 years. 